\title{
Deep Learning Methods for Flood Mapping: A Review of Existing Applications and Future Research Directions
}

\author{
Roberto Bentivoglio ${ }^{1}$, Elvin Isufi ${ }^{2}$, Sebastian Nicolaas Jonkman ${ }^{3}$, and Riccardo Taormina ${ }^{1}$ \\ ${ }^{1}$ Department of Water Management, Faculty of Civil Engineering and Geosciences, Delft University of Technology \\ ${ }^{2}$ Department of Intelligent Systems, Faculty of Electrical Engineering, Mathematics and Computer Science, Delft University \\ of Technology \\ ${ }^{3}$ Department of Hydraulic Engineering, Faculty of Civil Engineering and Geosciences, Delft University of Technology \\ Correspondence: Roberto Bentivoglio, r.bentivoglio@tudelft.nl
}

\begin{abstract}
Deep Learning techniques have been increasingly used in flood risk management to overcome the limitations of accurate, yet slow, numerical models, and to improve the results of traditional methods for flood mapping. In this paper, we review 45 recent publications to outline the state-of-the-art of the field, identify knowledge gaps, and propose future research directions. The review focuses on the type of deep learning models used for various flood mapping applications, the flood types considered, the spatial scale of the studied events, and the data used for model development. The results show that models based on convolutional layers are usually more accurate as they leverage inductive biases to better process the spatial characteristics of the flooding events. Traditional models based on fully-connected layers, instead, provide accurate results when coupled with other statistical models. Deep learning models showed increased accuracy when compared to traditional approaches and increased speed when compared to numerical methods. While there exist several applications in flood susceptibility, inundation, and hazard mapping, more work is needed to understand how deep learning can assist real-time flood warning during an emergency, and how it can be employed to estimate flood risk. A major challenge lies in developing deep learning models that can generalize to unseen case studies and sites. Furthermore, all reviewed models and their outputs, are deterministic, with limited considerations for uncertainties in outcomes and probabilistic predictions. The authors argue that these identified gaps can be addressed by exploiting recent fundamental advancements in deep learning or by taking inspiration from developments in other applied areas. Models based on graph neural networks and neural operators can work with arbitrarily structured data and thus should be capable of generalizing across different case studies and could account for complex interactions with the natural and built environment. Neural operators can also speed up numerical models while preserving the underlying physical equations and could thus be used for reliable real-time warning. Similarly, probabilistic models can be built by resorting to Deep Gaussian Processes.
\end{abstract}

\section{Introduction}

Flooding is one of the most dangerous and frequent natural hazards, accounting for significant human and economic losses every year (Jonkman and Vrijling, 2008). Because of the climate change effects, more frequent and intense extreme precipitations are expected to further increase the severity of this hazard (Masson-Delmotte et al., 2021). To mitigate the impact of 
floods on human lives and properties, both preventive and emergency measures are required (European Union, 2007). Preventive measures are operations aiming at reducing the possibility of a certain area being flooded. Those can be determined by maps that indicate the hazard of floods, i.e., the potential flood characteristics for an event. Instead, emergency measures are operations carried out just before, during, or after a flooding event. In those cases, real-time knowledge of the extent of the flood and the areas in danger is needed to execute countermeasures (Lendering et al., 2016).

There are three main flood maps used for dealing with such measures: (i) susceptibility maps provide a qualitative categorization of the flood hazard in an area given its physical characteristics and are used for preventive measures; (ii) flood extent or inundation maps determine the observed inundation extent, during or after the event, and are used for emergency measures; (iii) flood hazard maps indicate the spatial distribution of variables that characterize the flood hazard of a specific event, such as flood depth and water extent, and are used for both preventive and emergency measures. Traditionally, susceptibility maps are obtained with multi-criteria decision analysis (MCDA) (e.g., Abdullah et al., 2021), inundation maps with remote sensing analysis (e.g., Lin et al., 2016), and hazard maps with numerical methods (e.g., Dottori et al., 2021). Despite their wide usability, each method has its limitations. MCDA for flood susceptibility is simple and interpretable but its results are not accurate for complex phenomena (Khosravi et al., 2020). Moreover, the weights assigned to each criterion are subjective and thus biased by the external choices. Remote sensing analysis for flood inundation requires manual or semi-automated procedures to improve the results and additional data such as land cover distribution (e.g., Manavalan, 2017). In addition, traditional models for flood inundation are not scalable to large amounts of data, as the ones currently produced by worldwide satellite missions. Numerical methods for flood hazard modeling are robust and effective, but fast and accurate flood simulations remain a challenge (Costabile et al., 2017). There exist several ways to improve the speed of the simulations, for example, through parallel computing (e.g., Zhang et al., 2014; Ming et al., 2020; Glenis et al., 2013), or simplified models (e.g., Zhao et al., 2021a; Sridharan et al., 2021). However, parallel computing has high computational costs and simplified models are unable to correctly reproduce rapidly-evolving flows such as in urban floods (Costabile et al., 2017) and dam breaks (Prestininzi, 2008). Moreover, numerical models have intrinsic limitations which depend on the discretization of the governing physical equations and physical domain.

To overcome these limitations, practitioners and developers have used data-driven models based on machine learning. Machine learning (ML) is a branch of artificial intelligence in which the model improves its performance, with respect to some class of tasks, as the available data increases (Mitchell, 1997). Conventional ML techniques require specific feature engineering of raw data before its processing. Deep learning (DL) can, instead, automatically discover the representations needed for detection or classification in raw data (LeCun et al., 2015). DL methods are representation-learning methods with multiple levels of representation, obtained by composing simple but non-linear modules that each transform the representation at one level (starting with the raw input) into a representation at a higher and more abstract level (LeCun et al., 2015). The model can then learn hidden patterns in the data and, consequently, improve its performance. Both ML and DL models have been applied in the fields of hydraulics and flood analysis. Mosavi et al. (2018) examined ML models for the prediction of floods, in the short and long term. Sit et al. (2020) reviewed deep learning models for hydrology and water resources, focusing also on the 
hydrological modeling of floods. Zounemat-Kermani et al. (2020) reviewed neurocomputing for surface water hydrology and hydraulics including some applications concerning floods.

The existing reviews mainly focused on the temporal variability of floods, especially concerning rainfall-runoff modeling, covering only a few instances of flood mapping applications. However, the spatial evolution of flood events is extremely important to determine affected areas, plan mitigation measures and inform response strategies. The research community has put significant efforts in developing DL approaches to advance data-driven flood mapping and replace computationally expensive numerical models. The aim of this review is thus to advance the emerging field of DL-based flood mapping by surveying the state-of-the-art, identifying outstanding research gaps and proposing fruitful research directions.

45 papers are analysed considering two main parallel yet intertwined directions. On the one hand, we focused on the flood management application, spatial scale of study, and type of flood. On the other hand, we examined the deep learning model, type of training data, and performance with respect to alternative methods. This strategy provides insights from a flood management perspective and concurrently facilitates reflection on how to successfully apply DL models. For each category, we identify common patterns and deduce considerations based on the presented results, while also identifying papers presenting innovative approaches. Moreover, we compare against traditional methods to further validate the benefits of employing DL models. Finally, we highlight a series of current knowledge gaps and propose possible solutions to them, by drawing parallelisms with the recent advances in deep learning.

The remaining of this review is organized as follows. In Section 2, we present the background theory on both floods and deep learning. Then, in Section 3, we present the search methodology and then illustrate and discuss the findings of the review. In Section 3, we discuss the results based on the reviewed papers. In Sections 4 and 5 we present the knowledge gaps and propose possible future research directions. Finally, conclusions are provided in Section 6.

\section{Background}

This section is divided in two parts: flood management and deep learning. In the first part, we present the categories in which we classify flood management, while in the latter we describe the main deep learning models used for flood mapping.

\subsection{Flood management}

Floods can be defined as an overflow of water in otherwise dry land. Hence, flood management is a very broad field of interest: wherever there is water, there is a certain probability of being affected by it. While there exist several categorizations of flood management, we focus on types of floods, applications, and spatial scales.

\subsubsection{Types of floods}

We can distinguish flooding depending on how, why, and when it occurs:

- River floods are caused by extensive precipitation over long periods, causing the river to overflow its banks, ultimately inundating the neighboring areas. This process is slow and can last for several days (Serinaldi et al., 2018). 
- Flash floods are caused by short but intense rainfall or sudden melting of snow (Sikorska et al., 2015). They are rapid and intense floods, typical of mountain and steep catchments. Flash floods are usually coupled with other hazards such as debris flows (Destro et al., 2018) and landslides (Ávila et al., 2016).

- Coastal floods are caused by extreme meteorological conditions, which increase the water level in large bodies of water, due to a combination of low atmospheric pressure and strong winds. They occur near oceans, seas, or large lakes and we include in this category also tsunamis, although they are generated by geological phenomena such as earthquakes.

- Urban floods are caused by the failure of drainage from a sewer system, due to extreme precipitation, resulting in the overflow of those pipes. Depending on the city position and topography, these floods can also be affected by all the other types of floods.

- Dam and dike breach floods are caused by the failure of flood protection structures, due to extreme flood events or management issues. The uncertainty in if, where and how a defence will fail further increase the unexpectedness of these phenomena.

To simplify the categorization, we excluded pluvial flooding, i.e., floods caused by the failure of a drainage system due to intensive precipitation. The underlying hypothesis is that pluvial floods can be addressed as urban floods in urban environments or river floods if they also feature rainfall-driven river overflows. We also excluded from the review dam and dike breach floods since no paper analyzed them with deep learning.

\subsubsection{Flood mapping applications}

Since we focus on the spatial variability of floods, we distinguish among three types of mapping: flood susceptibility, flood inundation, and flood hazard.

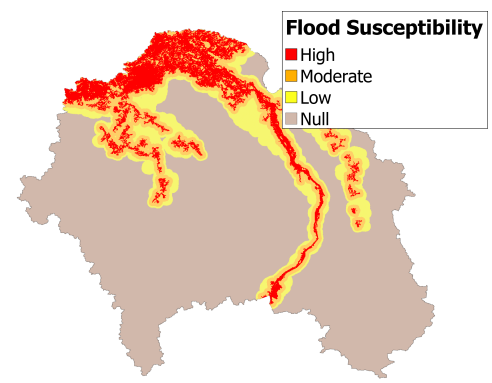

(a) Flood susceptibility

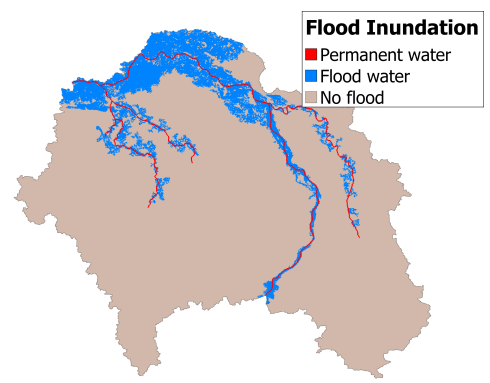

(b) Flood inundation

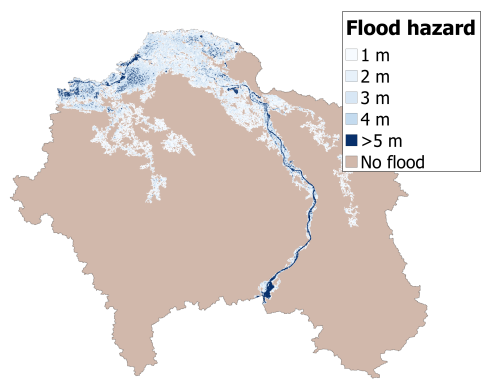

(c) Flood hazard

Figure 1. Examples of the types of flood maps analyzed for a hypothetical area: (a) shows a possible flood susceptibility map; (b) a flood inundation map; and (c) a flood hazard map, as defined in this paper.

- Flood susceptibility maps determine the tendency to flooding of a study area based on its physical characteristics (see Fig. 1a). This measure is only qualitative and does not evaluate any flood variable. Flood susceptibility mapping is 
performed by considering topographical, geographical, and meteorological factors (such as altitude, slope, lithology, land use, and rainfall) and comparing their spatial distribution with past flood events. This is done with multivariate analysis (e.g., Tehrany et al., 2014; Youssef et al., 2016) and multi-criteria decision analysis (e.g., Kazakis et al., 2015; Mahmoud and Gan, 2018). This analysis is carried out at large scales to assess areas at risk for spatial planning and flood mitigation strategies;

- Flood inundation maps determine the extent of a flood, during or after it has occurred (see Fig. 1b). Flood inundation maps represent flooded and non-flooded areas. This application is used for post-flood evacuation and protection planning, and for damage assessment. Flood images are obtained through remote-sensing techniques and processed by histogrambased models (e.g., Martinis et al., 2009; Manjusree et al., 2012), threshold models (e.g., Cian et al., 2018), and machine learning models (e.g., Hess et al., 1995; Ireland et al., 2015).

- Flood hazard maps measure the water depth and extent across a flooded area (see Fig. 1c). Hazard maps consider also different return periods of the floods and, thus, the probability of a certain event. The latter is determined through a statistical analysis based on the frequency and intensity of floods (Bobée and Rasmussen, 1995). We will refer to flood hazard also when the water depths are estimated, independently of the return periods. Flood hazard can also provide a measure of the flow velocities. Flood hazard maps are carried out by numerical models, which simulate flood events by discretizing the governing equations and the computational domain. Several numerical models were proposed in the last three decades (e.g., Horritt and Bates, 2002; Teng et al., 2017). One-dimensional (1D) models represent a channel through a mono-dimensional curvilinear axis (Cunge et al., 1980). They are fast and provide accurate results for the channel, but are less for floodplains outside the channel (Tayefi et al., 2007). Two-dimensional (2D) models represent the computational domain by meshes (e.g., Alcrudo and Garcia-Navarro, 1993). Mesh-based finite volume methods are the most used in practice (e.g., Dottori et al., 2021). Those methods give more accurate results than 1D models, but pay in longer computational time (Costabile et al., 2015). To increase the speed, the governing equations can be simplified by either approximating the models with less terms (Bates and De Roo, 2000), or by substituting these models with a simple sets of rules (Dottori and Todini, 2010). There are also 1D-2D coupled models connecting a 1D domain to a 2D domain (e.g., Finaud-Guyot et al., 2011) and are used for urban floods (Leandro et al., 2009) or to include 1D longitudinal elements in 2D models. Three-dimensional (3D) models represent the domain with meshes (Lane et al., 1999) or particles (Vacondio et al., 2012). They are particularly useful for exceptional events such as tsunamis and dam breaks. However, they are computationally demanding and thus less used than the other models.

Flood damage and flood risk maps (de Moel et al., 2009) are other examples of mapping applications. However, they are not described in more details here as no related DL-based paper was found in the literature. Similarly, the review also excludes applications which do not result in maps, such as water level forecast. 


\subsubsection{Spatial scale}

The importance of flood processes and the resolution of the flood maps varies with their spatial scale. Following de Moel et al. (2015), we also distinguish between local, regional, national, and supra-national scales. The choice between scales is often subjective, but here follows a rational categorization:

- Local scale refers to small study areas, such as towns or a specific river stretch. If a measure of the study area is given, we consider it in this category if the area is smaller than $<100 \mathrm{~km}^{2}$.

- Regional scale considers a specific province, watershed or large city. Study areas smaller than $<50000 \mathrm{~km}^{2}$ belong to this scale.

- National scale refers to assessments of entire countries, for which consistent (national) data are present. To exclude small countries, the study area must be greater than $<50000 \mathrm{~km}^{2}$.

- Supra-national scales concern assessments of an entire continent or the globe.

\subsection{Deep learning}

Deep learning studies how neural networks learn representations from data through multiple levels of abstraction. A neural network is a non-linear compositional model formed by a hierarchical layering of parametric functions that take an input variable $x$ and produce an estimate $\hat{y}$ of a target representation $y$ as $\hat{y}=f(x ; \theta)$, where $\theta$ are the function's parameters. The raw data $x$ are input to the neural network and the output of each layer serves as input for the following layer, until the final layer, which coincides with the estimate $\hat{y}$. A neural network with $L$ layers can be expressed as

$$
\begin{aligned}
\hat{y} & =f_{L}\left(\cdot ; \theta_{L}\right) \circ f_{L-1}\left(\cdot ; \theta_{L-1}\right) \circ \ldots \circ f_{1}\left(x ; \theta_{1}\right), \\
x_{\ell} & =f_{\ell}\left(x_{\ell-1} ; \theta_{\ell}\right), \text { for } \ell=1, \ldots, L, \\
\hat{y} & \equiv x_{L},
\end{aligned}
$$

where $f_{\ell}\left(\cdot ; \theta_{\ell}\right)$ is the function at layer $\ell$, ० represents the composition of functions, $\theta_{\ell}$ are the trainable parameters, and $\hat{y}_{\ell}$ is the output layer $\ell$. In a network architecture, the layers between the input and the output layer are called hidden layers, since their output is not shown. Estimating parameters $\theta_{\ell}$ is typically referred to as 'learning' and it is performed by minimizing a loss function, through back-propagation (Rumelhart et al., 1986). Depending on the task, neural networks can be trained via supervised and unsupervised learning. Since in flooding analysis DL has been mainly approached via supervised learning, we focus on that learning process.

Supervised deep learning models identify a mapping from input to output, given a training set of input-output pairs. For example, a training set for flood hazard comprises a flood's rainfall hyetograph as input $x$ and the corresponding maximum flooded area as output $y$. Thus, the loss function $l(y, \hat{y})$ compares the real output $y$ with the predicted one $\hat{y}$. The loss function is typically the quadratic loss for regression problems, where the data is continuous (e.g., water depth), or the cross-entropy 
loss for classification problems, where the data is categorical (e.g., flooded and non-flooded areas). As training data, we can have observations or simulations. Observational data are derived from remote sensing, flood inventory maps, and measuring stations, while simulation data are derived from numerical solvers. Once a model is trained, its goodness of fit is analyzed with a test set, composed of data that the model has not seen. If the model performs well for the test set, it is said to generalize or extrapolate well. The ability to generalize is one of the most important properties of DL and becomes even more important in high-dimensional inputs (Balestriero et al., 2021).

\subsubsection{Fully-connected layers}

Among the possible neural network layers, fully-connected ones are the most simple. In a fully-connected layer, the layer propagation rule is given by:

$x_{\ell}=f_{\ell}\left(x_{\ell-1}, \theta_{\ell}\right)=\sigma\left(W_{\ell} x_{\ell-1}\right)$,

where $x_{\ell}$ is the output of the layer $\ell, \sigma(\cdot)$ is a point-wise nonlinearity (e.g., $\operatorname{ReLU}, \sigma(x)=\max \{0, x\}$, or $\operatorname{Sigmoid}, \sigma(x)=$ $\left.\frac{1}{1+e^{-x}}\right), x_{\ell-1}$ is the input of the layer $\ell$, and the training parameter $W_{\ell}$ is a weight matrix. Multi-layer perceptrons (MLP) are composed by sequences of fully-connected layers (Fig. 2a). The dimensions of the hidden layers increase to increase the expressivity of the network, as shown in Fig. 2a. When the dimension of the hidden layers decreases and then increases, as shown in Fig. 2b, the architecture is called encoder-decoder (ED). The idea behind this architecture is that only certain latent representations of the input are useful to represent the output (e.g., Taormina and Galelli, 2018).

In fully-connected layers, the values of the parameters in $W$ are independent between them and there is no reuse of any of them. Thus, the number of learnable parameters is of the order of the input size, making fully-connected layers inappropriate for inputs of large dimensions. This issue is referred to as the 'curse of dimensionality' and implies that as the dimension of the input increases, the amount of training data needed to learn representations increases exponentially (LeCun et al., 2015).

To overcome the curse of dimensionality we need to exploit the structure in data. In flood analysis, data is usually structured: for example, neighboring pixels in raster data represent spatial proximity of nearby close elements, while discharge values in a hydrograph represent temporal proximities. Neural network layers can thus be defined in a way to exploit these data structures. These assumptions create what is known as an inductive bias, which imposes constraints on relationships and interactions among inputs in the learning process, thus prioritizing some solutions over others (Battaglia et al., 2018), as shown in Table 1. Inductive biases derive from two fundamental geometric principles, symmetry and scale separation (Bronstein et al., 2021). The symmetry of a system is a transformation that leaves a certain property of said system unchanged. Symmetry results in invariance and equivariance properties. Invariance implies that transformations on the input features do not change the output (i.e., $f(g(x))=f(x), g(\cdot)$ being a generic transformation), while equivariance entails that transformations on the input features change the output via an equivalent transformation (i.e., $f(g(x))=g^{\prime}(f(x)), g^{\prime}(\cdot)$ being a transformation equivalent to $g(\cdot))$. Scale separation, instead, denotes that localized interactions can be propagated towards coarser scales. Thus, the model can exploit local patterns even when considering large inputs. Inductive biases thus lead to the reuse of parameters in different parts of the input of each layer. For instance, convolutional kernels can be used on images of different dimensions and recurrent 


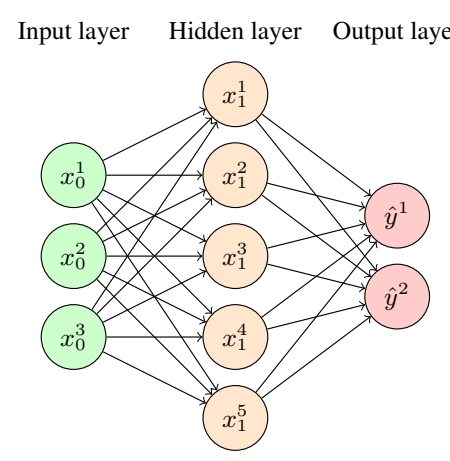

(a) Multi-layer perceptron

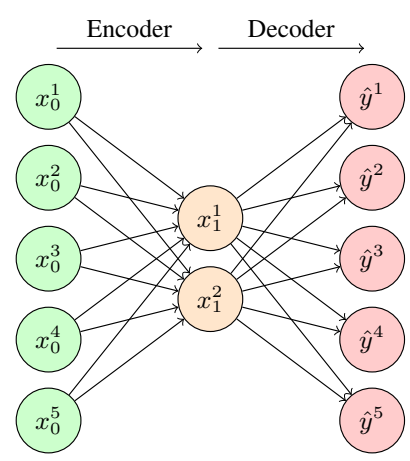

(b) Encoder-decoder

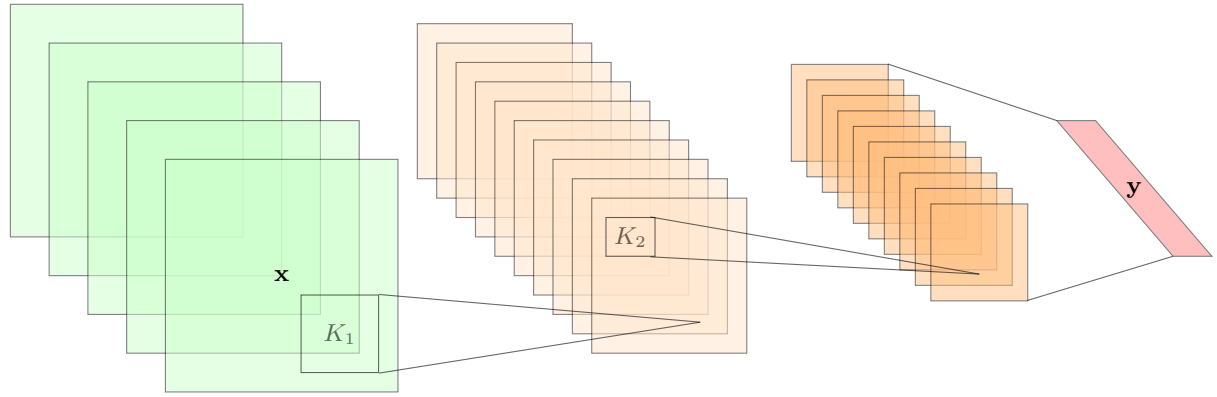

(c) Convolutional neural network

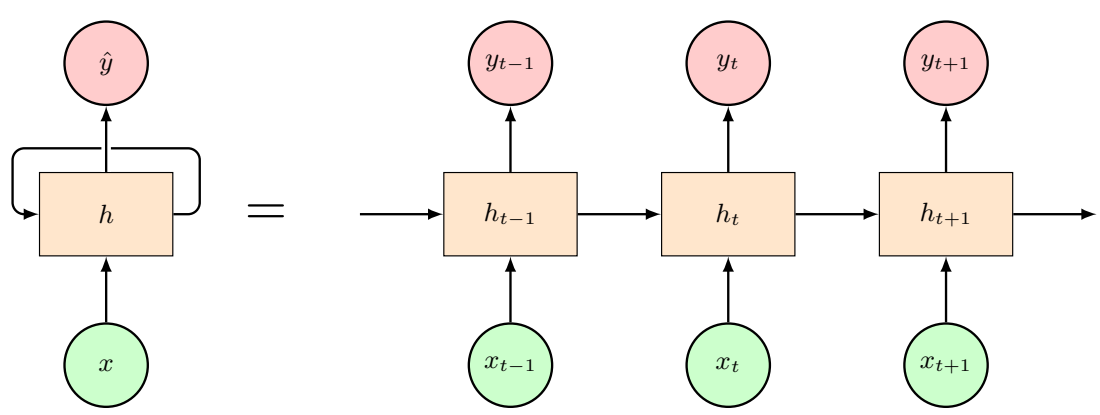

(d) Recurrent neural network

Figure 2. Deep learning architectures: (a) a multi-layer perceptron (MLP) composed of a sequence of three fully-connected layers. Every layer is connected to the following one by weights, represented by directed arrows. The values of the input, hidden, and output layers are represented, respectively, by vectors $x_{0}, x_{1}$, and $\hat{y}$; (b) an MLP encoder-decoder. The input data $x_{0}$ is encoded into a lower dimensional layer $x_{1}$, and then decoded into the output $\hat{y}$. This structure is also applicable to convolutional and recurrent layers; (c) a convolutional neural network $(\mathrm{CNN})$ composed of a convolutional layer, a pooling layer, and a fully-connected layer. The green squares represent an input tensor, the orange squares represent hidden layers and the red parallelogram on the right represents the output layer. The small box $K_{1}$ represents the convolutional kernel described in Eq. 3. The final layer depends on the task; (d) a recurrent neural network (RNN) in compact form (left) and in the unfolded form (right). The iterative structure of the RNN (left) can be unfolded in time to show how hidden states influence the solution at each time step (right). The colouring scheme indicates for each architecture the input (green), the state (orange), and the output (red). 
layers can consider time series of variable length. Fully-connected, instead, cannot have such inductive bias capabilities. The main characteristics for each considered layer are synthesized in Table 1. The input data type and the inductive biases are described for each studied layer.

Table 1. Inductive biases and preferred types of data for different neural network layers (adapted from Battaglia et al. (2018)).

\begin{tabular}{l|c|c}
\hline Layer & Data type & Inductive bias \\
\hline Fully Connected & Unstructured data & - \\
Convolutional & Grid elements & Spatial equivariance \\
Recurrent & Sequences & Temporal equivariance \\
\hline
\end{tabular}

\subsubsection{Convolutional layers}

Convolutional layers convolve the input with a trainable kernel (LeCun et al., 1995). A kernel is a filter which is applied to the input and learned during training. The propagation rule of layer $\ell$ of a convolutional layer is

$x_{\ell+1}=\sigma\left(K_{\ell} * x_{\ell}\right)$,

where $K_{\ell}$ is the kernel function for the $\ell^{\text {th }}$ layer and $*$ is the convolution operator. Convolutional layers are mostly applied to images i.e., two dimensional spatial grids. For such inputs the kernel is a matrix. Convolutional layers have an inductive bias of translational equivariance, which reflects the idea that spatially close grid elements influence each other. This results in the reuse of the same kernel across the different input parts and it implies that it matters where the pattern (object) is in an image and that the model should be able to recognize it. Convolutional layers thus perform feature extraction, identifying relevant characteristics in the input. Moreover, the reuse of parameters allows inductive learning over images of different sizes or resolutions. Differently from fully-connected layers, the number of parameters in a convolutional layer depends only on the kernel size because of this parameter sharing properties (see Fig. 2c). Depending on the input dimensions, we distinguish 1D convolutional layer for vector inputs, such as a rainfall hyetograph, 2D convolutional layers for matrix inputs, such as a digital elevation model (DEM), and 3D convolutional layers for tensor inputs, such as stacked satellite images. Since 1D-convolution considers translation equivariance on vectors, the inductive bias is equivalent to temporal equivariance if the vector is a time series.

Convolutional neural networks $(\mathrm{CNN})$ are composed of layers alternating convolution and pooling. Pooling operation replaces the output at a certain location with a summary statistic of the nearby features, thus reflecting translational invariance (Bronstein et al., 2021). They extract a single feature, such as the average or maximum value in a certain neighborhood of a point. Furthermore, pooling reduces the dimension of the input speeding up computation. The final layers of a CNN are typically fully connected when dealing with classification or regression tasks. This layer allows to map the convolved embeddings to the number of classes or to the regressed value, respectively. Instead, if the task is to perform image segmentation, 
the final layers are composed of de-convolutional layers which perform an operation opposite of convolutional layers, in an encoder-decoder structure. For details on convolutional layers and CNNs refer to Goodfellow et al. (2016).

\subsubsection{Recurrent layers}

Recurrent layers are used for processing sequential data, such as time series (Rumelhart et al., 1986). A recurrent layer can be seen as a nonlinear state-space model expressing the output at time $t, y_{t}$, as a function of a former hidden state $h_{t}$ and input $x_{t}$. The basic formulation for a recurrent layer is

$h_{t}=\sigma\left(W h_{t-1}+U x_{t}\right)$,

$y_{t}=\sigma\left(V h_{t}\right)$,

where $U, V$, and $W$ are trainable weight matrices. As it follows from (4), the hidden state encodes the temporal memory of previous time instances while the output mapping is instantaneous. These matrices are shared across time allowing the recurrent layer to exploit temporal proximities of sequential data, irrespectively of their position. This is for instance the case of discharge hydrographs (e.g., Zhou et al., 2021). Because there is an inductive bias in temporal sequences, they allow us to reuse parameters without affecting the performance.

Recurrent neural networks (RNN) are neural networks composed of recurrent layers. The iterative structure of the RNNs can be unfolded in time to show how hidden states influence the output at each time step (Fig. 2d). However, the vanilla recurrent layer in (4) suffers from the problem of vanishing and exploding gradients (Hochreiter and Schmidhuber, 1997). This occurs due to the iterative use of the same layer which causes the weights to multiply several times when back-propagating the error, ultimately leading to "vanishing" gradients if the weights are small and "exploding" gradients if the weights are large. This constrains then the temporal memory of these networks and limits their capability to extract long-term dependencies between the past inputs and the current output.

This problem is typically solved via the use of Long Short-Term Memory (LSTM) layers (Hochreiter and Schmidhuber, 1997). This variation of recurrent layers also improves the hidden state mechanism allowing to "remember" well even information which is temporally distant. Another common variation is the Gated Recurrent Unit (GRU) (Cho et al., 2014), which achieves comparable results with the LSTM architecture while using a simpler formulation. Same as for fully-connected and convolutional layers, recurrent layers can be used in encoder-decoder architectures. This structure can be composed of an RNN which generates a latent representation, followed by another RNN that decodes it.

The most successful applications of RNNs related to flood management regard tasks related to sequences and time-series analysis, such as rainfall-runoff modeling (Kratzert et al., 2019a). While RNNs are preferred over 1D-CNNs, recently the latter started gaining momentum for some time-series learning tasks (e.g., Oord et al., 2016). 


\section{Review}

\subsection{Methodology}

Papers were retrieved from the Scopus database by combining the keywords "deep learning" or "neural network" with "flood" or "flooding". The 3017 publications obtained were then filtered to include only journal papers from 2010 onward, in the areas of engineering, environmental science, and earth and planetary sciences. From this reduced list of 1088 papers, we considered two major refining criteria: i) the papers should be based on the deep learning models presented in Section 2.2, and ii) the applications must address the spatial variability of floods (e.g., not focusing only the temporal aspects of flood analysis). This procedure resulted in 35 reviewable papers. This list was finally extended via a snowball search that considered cited and citing works, ultimately leading to 45 eligible documents. We find that the described methodology selected a representative subset for producing a thorough review of recent advances and developments in this field.

The selected papers are listed in Table 2 which reports major details including the flood mapping application, the type of flood, the DL model, and the spatial scale. General findings related to these three criteria are first presented in Section 3.2. Specific findings for each application are then presented in Sections 3.3 (flood susceptibility), 3.4 (flood inundation), and 3.5 (flood hazard). These specific sections provide a more in-depth discussion on the deep learning models employed, with a focus on the architecture, the input and output data, and the performance assessment.

Table 2: Deep learning applications for flood mapping. References are classified in terms of flood mapping application, type of flood, deep learning (DL) model, training data, and spatial scale.

\begin{tabular}{|c|c|c|c|c|c|}
\hline Application & Flood Type & DL Model & Training Data & Spatial Scale & Reference(s) \\
\hline \multirow[t]{10}{*}{ Susceptibility } & River & MLP & Observations & Local & Khoirunisa et al. (2021) \\
\hline & & & & Regional & $\begin{array}{l}\text { Jahangir et al. (2019); Ahmadlou } \\
\text { et al. (2021); Popa et al. (2019); Kia } \\
\text { et al. (2012) }\end{array}$ \\
\hline & & $\mathrm{CNN}$ & Observations & Regional & Wang et al. (2020) \\
\hline & & & & National & Khosravi et al. (2020) \\
\hline & & RNN & Observations & Regional & Fang et al. (2020a) \\
\hline & Flash & MLP & Observations & Regional & $\begin{array}{l}\text { Tien et al. (2020); Ngo et al. (2018); } \\
\text { Popa et al. (2019); Costache et al. } \\
(2020)\end{array}$ \\
\hline & & & & National & Kourgialas and Karatzas (2017) \\
\hline & & $\mathrm{CNN}$ & Observations & Regional & Panahi et al. (2021) \\
\hline & Urban & MLP & Observations & Local & Darabi et al. (2021) \\
\hline & & & & Regional & Kalantar et al. (2021) \\
\hline
\end{tabular}


https://doi.org/10.5194/hess-2021-614

Preprint. Discussion started: 13 December 2021

(c) Author(s) 2021. CC BY 4.0 License.

(c) (1)
Hydrology and

Earth System

Sciences

Discussions

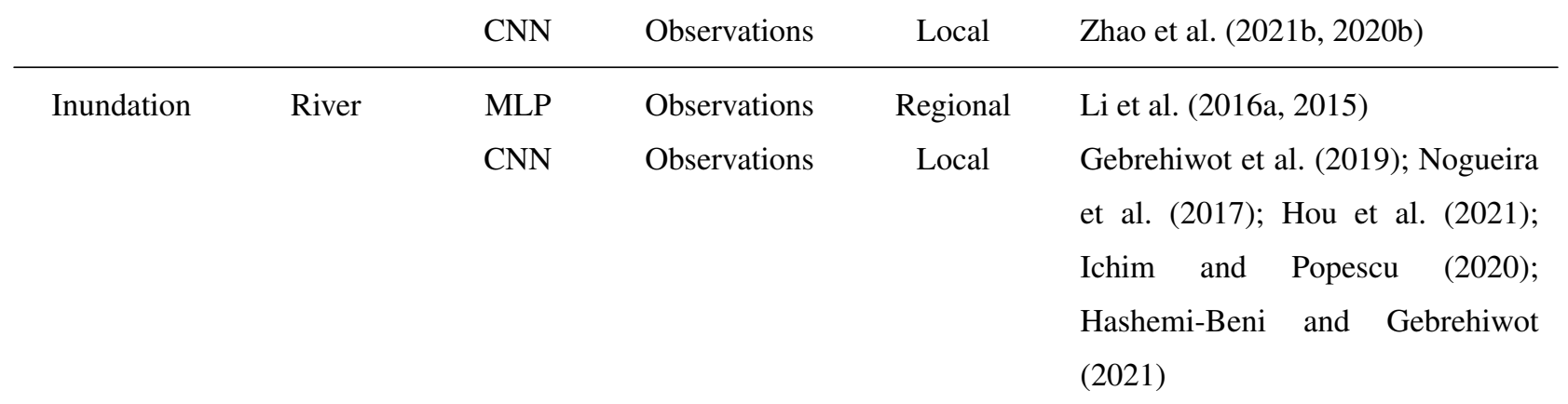

Regional Sarker et al. (2019); Kang et al. (2018); Nemni et al. (2020)

\begin{tabular}{|c|c|c|c|c|c|}
\hline & \multirow[t]{3}{*}{ Urban } & \multirow[t]{2}{*}{ MLP } & \multirow[t]{2}{*}{ Observations } & Local & Amini (2010) \\
\hline & & & & Regional & Li et al. (2016b) \\
\hline & & $\mathrm{CNN}$ & Observations & Local & Peng et al. (2019) \\
\hline & \multirow{3}{*}{ Coastal } & RNN, CNN & Observations & Local & Dong et al. (2021) \\
\hline & & $\mathrm{CNN}$ & Observations & Regional & Liu et al. (2019) \\
\hline & & & Simulations & Regional & Muñoz et al. (2021) \\
\hline \multirow[t]{7}{*}{ Hazard } & \multirow[t]{4}{*}{ River } & MLP & Simulations & Local & $\begin{array}{l}\text { Chu et al. (2020); Huang et al. } \\
\text { (2021a); Lin et al. (2020b, a) }\end{array}$ \\
\hline & & $\mathrm{CNN}$ & Simulations & Local & Kabir et al. (2020) \\
\hline & & & & Regional & Guo et al. (2020) \\
\hline & & RNN & Simulations & Regional & Zhou et al. (2021); Kao et al. (2021) \\
\hline & \multirow[t]{2}{*}{ Urban } & MLP & Simulations & Local & $\begin{array}{l}\text { Berkhahn et al. (2019); Chang et al. } \\
\text { (2010) }\end{array}$ \\
\hline & & $\mathrm{CNN}$ & Simulations & Regional & Löwe et al. (2021) \\
\hline & Coastal & RNN & Simulations & Local & Hu et al. (2019) \\
\hline
\end{tabular}

MLP=Multi-layer Perceptron; CNN=Convolutional Neural Network; RNN=Recurrent Neural Network

\subsection{General findings}

\subsubsection{Flood mapping applications}

Fig. 3 shows the distribution of papers for each of the applications considered: flood susceptibility, flood inundation, and flood hazard. The research community has dedicated efforts to investigate each type of application, although flood inundation and susceptibility have received the most attention. While papers on flood inundation are more evenly distributed across years, applications for flood susceptibility and, especially, flood hazard are increasing in the last few years. Similar to what was observed in related fields such as hydrology (e.g., Sit et al., 2020), a strong surge in DL publications for spatial flood analysis is 
https://doi.org/10.5194/hess-2021-614

Preprint. Discussion started: 13 December 2021

(c) Author(s) 2021. CC BY 4.0 License.

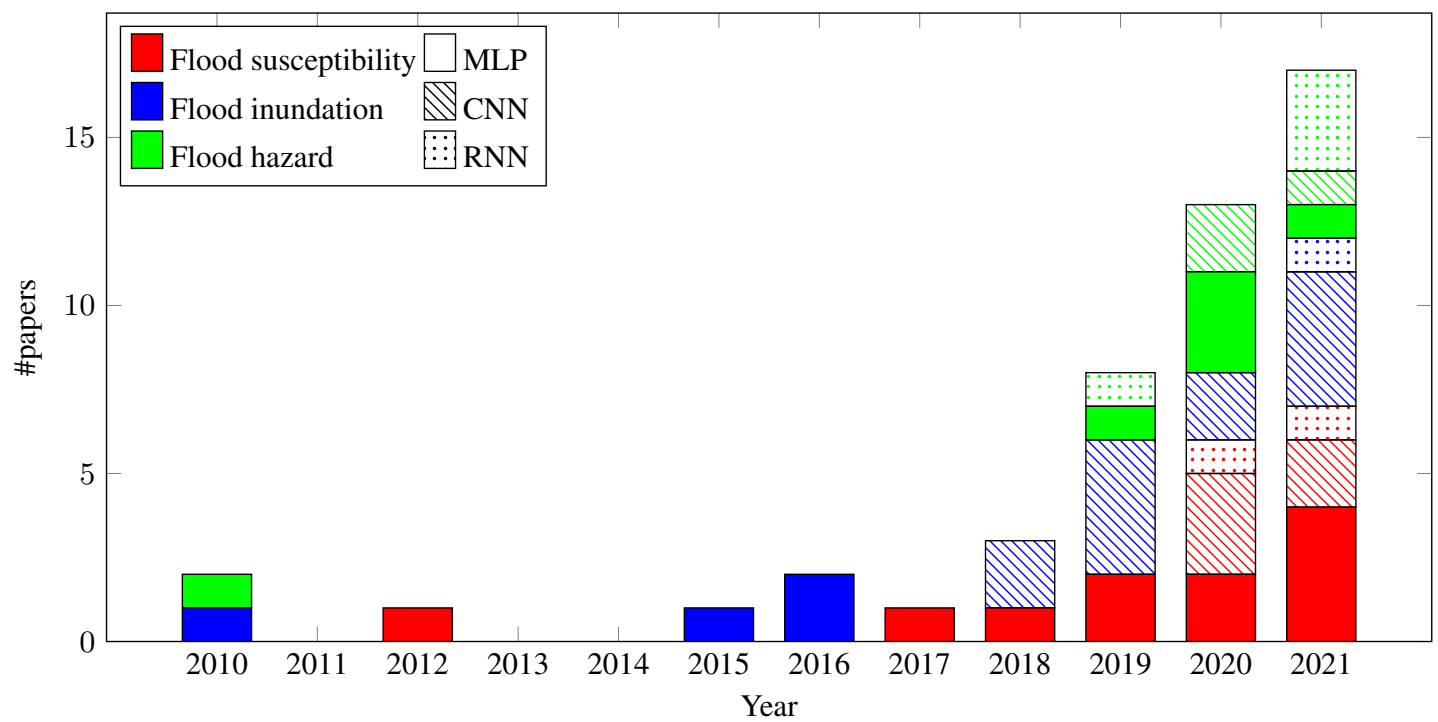

Figure 3. Publications by year, type of application and type of DL model. The increasing trend of the last five years has been mostly driven by the applications in flood susceptibility and flood hazard.

witnessed between 2018 and 2019. These years identify a turning point for AI in earth system sciences driven by the adoption of CNN (striped patterns in Fig. 3) and RNN (dotted patterns) in lieu of traditional MLP models.

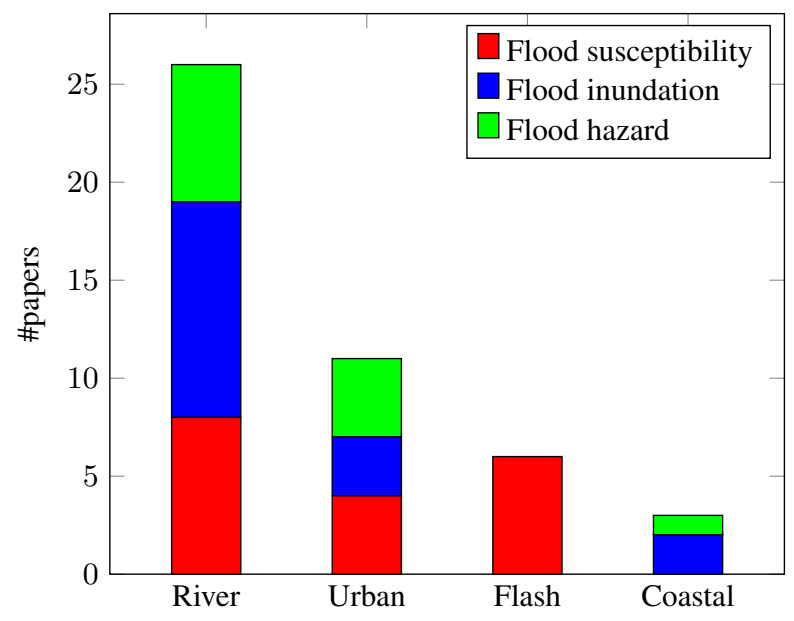

Figure 4. Distribution of the types of floods per flood application in the reviewed papers. River and urban floods are the most common, while flash and coastal floods have fewer occurrences. 


\subsubsection{Flood types}

Fig. 4 shows the types of flood analyzed with respect to each application. River floods are the most common, with many applications in inundation and hazard mapping. This is probably because, for historical reasons, most cities in the world are built close to rivers (Kummu et al., 2011). The scientific community has dedicated significant efforts to exploring the potential of DL for urban flooding. The latter is more difficult to model because of the complex topography and the presence of a drainage system whose dynamics need to be coupled with the overland flood (Löwe et al., 2021). All papers analyzing flash floods described flood susceptibility mapping applications. This is expected due to the short duration and the contingent nature of these phenomena, which limit remote sensing imaging and numerical simulations used in flood inundation and flood hazard mapping, respectively. Despite the importance of coastal flooding (Neumann et al., 2015), only a few papers report the use of DL for coastal flooding. While other works are available in the literature (Lütjens et al., 2020, 2021; Bowes et al., 2021), they were not considered since the employed DL models were not trained via supervised learning. Some of these works will be discussed in Section 5.

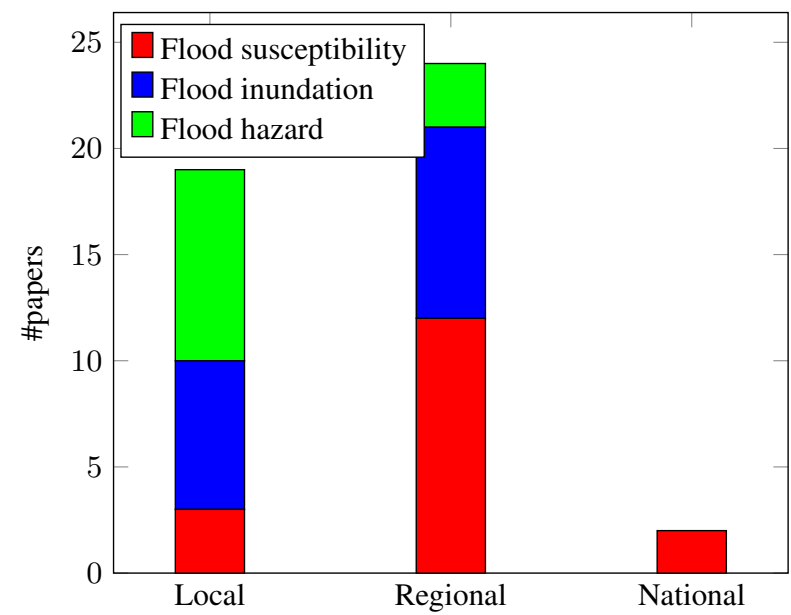

(a) Flood applications

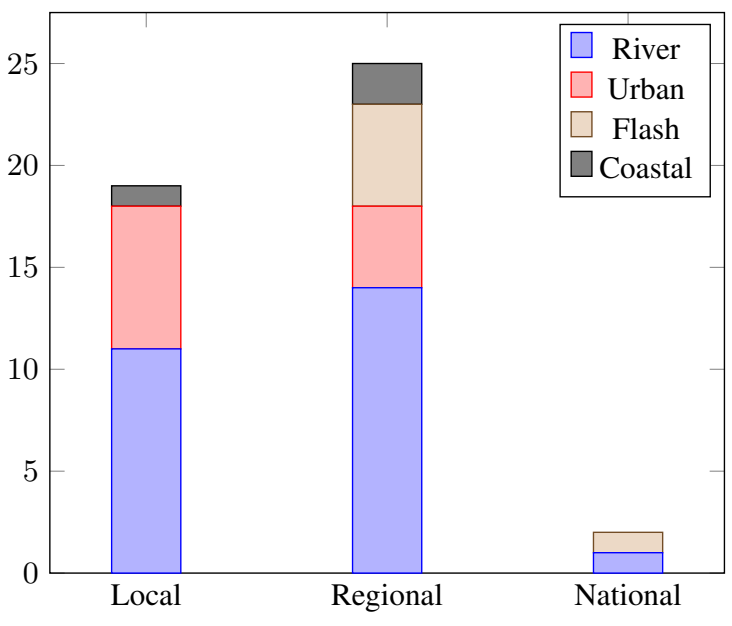

(b) Flood type

Figure 5. Distribution of the spatial scale per (a) flood application and (b) type of flood in the reviewed papers. Local and regional scales are the most used.

\subsubsection{Spatial scale}

As shown in Fig. 5a, most applications consider local and regional scales. Local scale refers to towns (e.g., Darabi et al., 2021;

Berkhahn et al., 2019), small catchments (e.g., Lin et al., 2020a; Kabir et al., 2020) or river reaches (e.g., Chu et al., 2020; Gebrehiwot et al., 2019). As such, they are mostly referred to urban and river floods. The cases sizes vary from very small ones, $10000 \mathrm{~m}^{2}$ (Gebrehiwot et al., 2019), to small towns up to $100 \mathrm{~km}^{2}$ (Lin et al., 2020a). Regional scale models consider a catchment (e.g., Popa et al., 2019), a province (e.g., Wang et al., 2020) or large cities (e.g., Löwe et al., 2021; Kalantar et al., 
2021). Most works focus on river floods, while some study flash, urban, and coastal floods. National scale models refer to

the assessments of entire countries with only two papers concerning such scales, respectively for Iran and Greece (Khosravi et al., 2020; Kourgialas and Karatzas, 2017). Nemni et al. (2020) and Sarker et al. (2019) consider several study areas across Africa and Asia, and Australia, respectively, but since the size of each area were smaller than $50000 \mathrm{~km}^{2}$ they were marked as regional scale models. They also do not fit within the national scale classification since they do not encompass whole nations. Supra-national scale models assessing the entire globe or a continent have not been studied yet with deep learning models. This seems unexpected since ML techniques have already been employed at global scales, outperforming traditional techniques, for example in the estimation of design floods along river networks (e.g., Zhao et al., 2020a). Since DL models have been shown to outperform ML models, as later outlined in this review as well, more models should be used at those scales in future studies.

\subsubsection{DL architecture}

Fig. 3 reports the architecture used for each application, showing that DL models are mainly based on fully connected and convolutional layers.

MLP networks are widely used due to their flexibility and ease of implementation. However, they are usually coupled with other techniques to reach satisfactory performances. Stochastic optimization techniques, such as genetic algorithm, firefly algorithm, and particle swarm optimization were combined with MLPs to search the optimal model's parameters (e.g., Li et al., 2015; Ngo et al., 2018; Kalantar et al., 2021). Multi-criteria decision analysis models, such as frequency ratio and analytical hierarchy process, were also coupled with MLPs to adjust the weights of each input in flood susceptibility (e.g., Kourgialas and Karatzas, 2017; Costache et al., 2020; Popa et al., 2019). Furthermore, k-means clustering was used to categorize the dataset in classes, to account for different topographical conditions; then, for each class, a MLP was trained (e.g., Chang et al., 2010; Huang et al., 2021a). Combining MLPs with such methods partly compensates the lack of inductive biases, however, this lack blocks the model from employing existing structures in the data, ultimately limiting their usability. Since flooding phenomena have spatial and temporal structures, we expect MLPs to become progressively less used in this field, as hinted by the trend in Fig. 3.

CNNs are best suited for processing raster files and images, thanks to their spatial inductive bias. Since most data for flood analysis (e.g., elevation data, rainfall distribution fields, remote sensing image) come in this format, CNNs have been increasingly employed by the research community in the recent years. While most papers consider standard CNNs, there are a few which employ 1D-CNNs (e.g., Dong et al., 2021; Guo et al., 2020) and 3D-CNNs (e.g., Wang et al., 2020; Fang et al., 2020a). 1D-CNNs consider as input a hyetograph or a hydrograph of a certain event, while 3D-CNNs consider raster files stacked upon each other. Regarding the architectures, different papers for flood inundation consider an encoder-decoder structure for image segmentation and classification (e.g., Nemni et al., 2020; Hashemi-Beni and Gebrehiwot, 2021; Liu et al., 2019). For such papers, the input is a satellite image of a flood and the output is its classification in flooded and non-flooded areas. This architecture allows the models to increase its performance since it can retain high frequency details in the segmented images (Badrinarayanan et al., 2015). 
Guo et al. (2020) and Löwe et al. (2021) use a convolutional encoder-decoder structure for flood hazard mapping to embed a rainfall hyetograph in the latent space. In this way, they can consider both spatial and temporal data within the same framework.

RNNs have been mostly employed to model temporally-varying floods, where they can exploit best their sequential inductive bias. However, they remain the least common choice of DL architecture for spatial flood analysis. Most papers apply RNNs on a time series, such as a hyetograph or a hydrograph (e.g., Kao et al., 2021; Zhou et al., 2021). Some papers, instead, consider spatial sequentiality by reshaping the original raster data into vectors (e.g., Fang et al., 2020a; Panahi et al., 2021). For example, Fang et al. (2020a) extract, for each pixel, its neighboring pixels in a $3 \times 3$ window and then convert them into a vector based on spatial contiguity. However, this operation introduces arbitrariety in the sequential order chosen for arranging the input pixels, since it is independent of the underlying topography. In fact, Panahi et al. (2021) shows that these models underperform when compared with CNNs. Among the different RNN layers, most works consider LSTM units (Kao et al., 2021; Zhou et al., 2021; Fang et al., 2020a) but simple recurrent units (Panahi et al., 2021; Huang et al., 2021a) and GRUs (Dong et al., 2021) have also been employed. Some papers analyzed the potential of RNNs in combination with other techniques. Kao et al. (2021) use an encoder-decoder architecture to forecast flood features based on rainfall patterns. The encoder and the decoder steps are composed of fully-connected layers, while a LSTM is present in the latent space to process rainfall data. Zhou et al. (2021) identify representative spatial locations in the study area. Then, an LSTM is trained to simulate the water levels' evolution in time at each location. A water surface is ultimately determined by interpolating the water depth at those points. Dong et al. (2021) combine 1D-CNNs and RNNs on an urban channel network. The model takes as input the channels' properties, such as their cross-sections, and rainfall and water level measures, taken from sensors in the network. This input is then given in parallel to a 1D-CNN and to a GRU whose output is then combined to predict the temporal evolution of the flood. Hu et al. (2019) deploy the LSTM model in a lower-dimensional space, obtained via proper orthogonal decomposition and singular value decomposition. The model then requires fewer data to be trained.

\subsubsection{Performance assessment}

This section discusses different approaches for assessing the performance of the DL models, i.e., how well they match the outcomes of traditional and machine learning models. Flood susceptibility and inundation models are compared with techniques such as frequency ratio (Popa et al., 2019), a type of MCDA model; the soil conservation service runoff model (Jahangir et al., 2019), a hydrologic model; and automatic threshold model (Nemni et al., 2020), a histogram-based model. They are also compared with machine learning techniques, such as support vector machines (e.g., Sarker et al., 2019; Gebrehiwot et al., 2019; Zhao et al., 2020b), random forest (e.g., Darabi et al., 2021; Zhao et al., 2020b), adaptive neuro-fuzzy inference system (Panahi et al., 2021), and radial basis function (Nogueira et al., 2017). DL models show to outperform both traditional and ML models in terms of the accuracy of the results. Flood hazard models, instead, are compared against numerical models, acting as surrogate models. Thus, their main purpose is to increase computational speed while maintaining low prediction errors.

There are also a few papers that compared different DL models. Huang et al. (2021b) compared MLPs with RNNs, while Fang et al. (2020a) showed that MLPs were outperformed by the more inductive-biased approaches such as RNNs, 1D-CNNs, and 3D-CNNs. There is still no comparison between CNNs and MLP. We expect CNNs to also outperform MLPs because of 
their inductive biases capabilities, as shown in other fields for similar tasks (e.g., Yi et al., 2020). Besides accuracy, the number of parameters and the data requirements are important factors when comparing DL models. A higher number of parameters results in better performances but may also lead to overfitting, a condition where the model decreases its performance on the testing data. Hence, when deployed in similar settings such model would perform drastically worse. Moreover, data is not always available leading to possibly unfair comparisons between models with different data budgets.

In supervised learning, we distinguish between regression and classification problems. Regression metrics deal with continuous data, such as water depths, and are computed from the difference between target and predicted values. The most common ones are the root mean squared error (RMSE), the coefficient of determination $\left(R^{2}\right)$, and the mean average error (MAE). RMSE and MAE improve as they approach zero, while $R^{2}$ improves as it approaches one. Categorical or classification metrics deal with discrete data, such as flooded and non-flooded areas, and determine which predictions are correct and which are misclassified. The most common ones are accuracy and area under the curve (AUC), but other indices such as the intersection over union (IoU) are seldom used as well. Accuracy represents the number of correct predictions over the total and its maximum is $100 \%$; the AUC describes how much a model can differentiate between classes, one being its maximum value; the IoU evaluates the overlapping between predicted and target classes, reaching a maximum value of $100 \%$. The classification can be either binary or multi-categorical, according to the output number of classes. Depending on the application, both types of metrics can be selected, as shown in Fig. 6. Further considerations are drawn for each application in the following sections.

For the surrogate models, the comparison is also performed in terms of their speed-up, which is determined as the ratio between the simulation time of the numerical model and the simulation time of the DL model. For a correct comparison, the training time of the DL model must be considered as well in this analysis. However, this was done only by a few papers (e.g., Guo et al., 2020; Kabir et al., 2020).

\subsection{Deep Learning for flood susceptibility}

Flood susceptibility determines the tendency to flooding of a study area based on its physical characteristics and given a set of known past flood events. This is done by assigning to each location a level of susceptibility ranked from low to high (see Fig. 1a). The susceptibility depends on the distribution of the inputs, often called flood conditioning factors, in function of recorded past flood events. The deep learning model then computes, for each point in the area, a score from 0 (non-flooded) to 1 (flooded). These scores are finally divided into several classes, generally using the natural (Jenks) breaks method (e.g., Fang et al., 2020a; Wang et al., 2020; Khoirunisa et al., 2021), to obtain a susceptibility map. An exception is given by Jahangir et al. (2019) and Kia et al. (2012), which train their models to predict discharge values and then use a GIS model for the mapping. In both cases, the model performs well when the recorded flood events occur in the predicted high susceptibility areas.

There exist DL-related applications for all types of flood apart from coastal floods (see Fig. 4). Furthermore, Fig. 5a shows that most of the works are concerned with regional or wider scales (e.g., Tien et al., 2020; Panahi et al., 2021; Khosravi et al., 2020). This is expected since susceptibility mapping gives a qualitative estimate of which locations are prone to flooding. Operating on small scales may thus be limiting, both in terms of data availability and applicability for prevention strategies. The data requirements for an accurate estimate would probably be too high for a small area. 
https://doi.org/10.5194/hess-2021-614

Preprint. Discussion started: 13 December 2021

(c) Author(s) 2021. CC BY 4.0 License.

(c) (1)
Hydrology and Earth System Sciences

Discussions

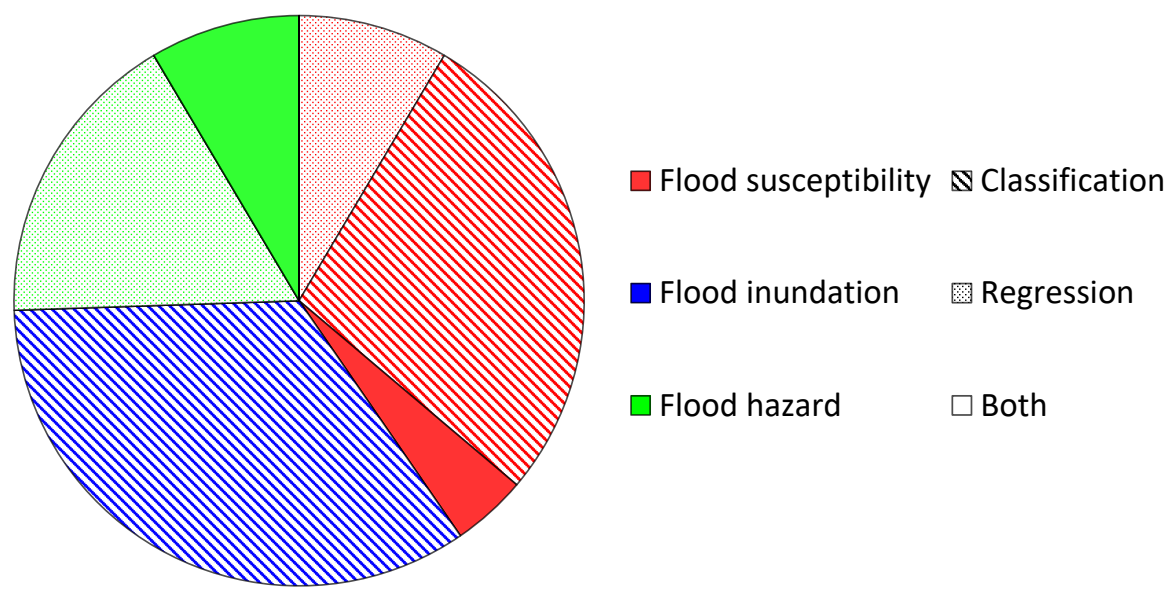

Figure 6. Distribution of the comparison metrics per type of application. The colours represent the different types of applications, while the patterns represent the considered metrics.

\subsubsection{DL architectures}

Most papers use MLP and CNN. Models based on MLPs consider single points or pixels as inputs (Tien et al., 2020; Ahmadlou et al., 2021; Khoirunisa et al., 2021), while CNNs consider the whole raster files (Zhao et al., 2020b; Khosravi et al., 2020; Wang et al., 2020). Since MLPs lack inductive bias they provide less coherent results, meaning that the variation among neighboring cells can be high. This is partially solved by coupling the MLP architecture with other statistical techniques, such as frequency ratio (e.g., Darabi et al., 2021; Popa et al., 2019; Costache et al., 2020). Since CNNs have a spatial inductive bias, they inherently consider the structure of the input, thus providing more coherent flood maps (e.g., Khosravi et al., 2020).

\subsubsection{Input and output data}

The inputs for the deep learning models are several, with an average of ten types. We distinguish between five input typologies:

1. topographical inputs, which are derived from a digital elevation model, such as elevation, slope, and aspect;

2. meteorological inputs, related to the hydrological characteristics and derived from measuring stations and satellites, such as rainfall distribution and frequency;

3. geological inputs, related to the properties of the soil, such as lithology and soil type; 
4. geographical inputs, related to observable surface characteristics and obtained through remote sensing, such as land use and normalized difference vegetation index;

5. anthropogenic inputs, related to the presence of human-made environments, such as distance from roads.

Topographical data were the most frequent type of input. Most papers also performed a statistical analysis to determine which factors influenced the most the final results: on average the most important factors were slope, land use, aspect, terrain curvature, and distance from the rivers (e.g., Khosravi et al., 2020; Fang et al., 2020a; Popa et al., 2019; Costache et al., 2020). A complete list of inputs is reported in the Appendix (Fig. A1).

As output data, most papers considered a flood inventory map, given by a set of flooded and non-flooded locations. The flooded locations were derived from measurements and records taken from remote sensing and stations, while non-flooded locations were taken randomly from locations with no previous flood record.

\subsubsection{Performance assessment}

In flood susceptibility analysis, both classification and regression metrics are adopted (Fig. 6). While classification metrics are used to identify flooded or non-flooded areas, the purpose of regression metrics is often omitted unless the reference target is a discharge hydrograph (Jahangir et al., 2019; Kia et al., 2012). Both types of metrics are used in few papers (Panahi et al., 2021; Khosravi et al., 2020). Because of the problem's setup, classification metrics are more reliable in the performance assessment. For all the papers with comparisons, deep learning models consistently showed improved performances with respect to the reference models (Table 3). Combining optimization algorithms, such as particle swarm optimization, with MLPs, to improve the training, has a limited effect on the performance improvement (Kalantar et al., 2021; Ngo et al., 2018). Moreover, CNNs increase the performance with respect to traditional models more than MLPs. Fang et al. (2020a) show that encoding spatial sequentiality with LSTMs work slightly better than 1D-CNNs and 3D-CNNs, however they avoid the comparison with 2DCNNs.

\subsection{Deep Learning for flood inundation}

435 Flood inundation maps determine the extent of a flood, during or after it has occurred. The objective is to determine flooded and non-flooded areas from a picture of a flood. This classification is usually binary (e.g., Peng et al., 2019; Nemni et al., 2020) but it can also be extended to include permanent water bodies (Sarker et al., 2019) (see the example Fig. 1b), vegetation (Ichim and Popescu, 2020), buildings (Hashemi-Beni and Gebrehiwot, 2021), and more (Muñoz et al., 2021). All the types of floods were well represented for this application but flash floods (Fig. 4). We attribute this to the limited frequency of observation of most remote sensing techniques.

Regarding the spatial scale, most papers focused on local and regional scales. Availability of remote sensing at wider scales is increasingly higher (e.g., Observatory); however, this seems to be only partially considered. A plausible reason is the limited frequency of observation of the satellites. High temporal remote sensing imagery has a low spatial resolution. Few papers tackle this issue by increasing the resolution of the predicted flood maps, via a neural network, with a technique known as super- 
Table 3. Comparison between deep learning and reference models for flood susceptibility.

\begin{tabular}{cccccl}
\hline $\begin{array}{c}\text { Case study } \\
\text { size }\left[\mathrm{km}^{2}\right]\end{array}$ & Model & Metric & Model & Metric & Reference \\
\hline 27 & MLP+ensemble & AUC $=0.847$ & RF & AUC $=0.821$ & Darabi et al. (2021) \\
147 & MLP+PSO & AUC $=0.98$ & MLP & AUC $=0.96$ & Kalantar et al. (2021) \\
207 & MLP & $R^{2}=0.82$ & SCS & $R^{2}=0.71$ & Jahangir et al. (2019) \\
1465 & MLP & Accuracy $=92 \%$ & SVM & Accuracy $=89.5 \%$ & Tien et al. (2020) \\
1510 & MLP+FA & Accuracy $=93.7 \%$ & SVM & Accuracy $=91.6 \%$ & Ngo et al. (2018) \\
5264 & MLP+FR & AUC $=0.97$ & FR & AUC $=0.937$ & Popa et al. (2019) \\
131 & CNN & Accuracy $=90 \%$ & RF & Accuracy $=86 \%$ & Zhao et al. (2020b) \\
1543 & CNN & AUC $=0.937$ & SVM & AUC $=0.883$ & Wang et al. (2020) \\
12000 & CNN & AUC $=0.832$ & ANFIS & AUC $=0.70$ & Panahi et al. (2021) \\
1543 & LSTM & Accuracy $=93.75 \%$ & $3 D-C N N$ & Accuracy $=92.19 \%$ & Fang et al. (2020a) \\
\hline
\end{tabular}

$\mathrm{PSO}=$ particle swarm optimization; SCS = soil conservation system model; FA = firefly algorithm; $\mathrm{SVM}=$ support vector machine; $\mathrm{RF}=$ random forest $\mathrm{FR}=$ frequency ratio; ANFIS = adaptive neuro-fuzzy inference system

resolution (e.g., Li et al., 2015, 2016b). Super-resolution enhances the quality of an input low-resolution image (Yang et al., 2019a). These papers show that MLPs improve the accuracy of super-resolution mapping, with respect to other techniques such as spatial attraction models. We argue that further improvements of super-resolution could be obtained by employing CNNs, which lend themselves naturally for such tasks, as demonstrated by applications in similar fields (Ma et al., 2019).

\subsubsection{DL architecture}

As the task of recognizing floods from a picture can be regarded as an image segmentation task, the most used deep learning models are based on CNNs. There are also a few earlier papers that use MLPs (e.g., Li et al., 2016a; Amini, 2010) because CNNs were not yet adopted by researchers of the field. Dong et al. (2021) use a combination of RNNs and 1D-CNNs to determine the temporal evolution of flooded and non-flooded nodes in an urban channel network, as described previously. In this case, the choice of recurrent and 1D-convolutional layers is well motivated due to their temporal inductive bias.

\subsubsection{Input and output data}

Satellite data is the most used input for flood inundation applications (e.g., Sarker et al., 2019; Peng et al., 2019; Nogueira et al., 2017). Other input data sources include unmanned aerial vehicles data (UAV) (e.g., Gebrehiwot et al., 2019; Ichim and Popescu, 2020), hydrographs (e.g., Hou et al., 2021) and DEMs (e.g., Hashemi-Beni and Gebrehiwot, 2021; Muñoz et al., 2021). Inundation maps produced by 3D numerical models are also used as target prediction (Muñoz et al., 2021). The results from the numerical model can be used as a detailed reference for the DL model. Satellite data and UAV imagery are both 
remote sensing data that represent a flood event seen from above. The main differences concern the scale, the resolution, and the availability. UAVs are applicable only for small areas but their resolution is better than satellite data. UAVs can be readily used but may be unavailable in certain areas. On the other hand, satellite data is available worldwide but its frequency of observation can be limiting. When combining information from different sources, the input data have different resolutions, leading to possible problems for some deep learning models, which take fixed-size inputs. One way to integrate different data resolutions is by data fusion (e.g., Muñoz et al., 2021). This process allows the creation of more consistent, accurate, and useful information than that provided by any individual data source.

\subsubsection{Performance assessment}

Since flood inundation mapping determines which cells of the flood picture are represented as flooded or not, the task is regarded as a classification problem, as confirmed by the metrics used (Fig. 6). Deep learning models have consistently shown improved performances in terms of accuracy and other metrics (Table 4). Li et al. (2015) and Li et al. (2016b) compare optimization techniques with and without MLPs for super-resolution-based flooding. They show that a DL model slightly increases the performances. This may be because the models are based on MLPs and thus neglect any spatial structure in the data, which could be considered, instead, by CNNs. Most CNN models show noticeable improvements with respect to traditional models, except for a few works with small improvements. This reflects similar results obtained in image detection tasks (Badrinarayanan et al., 2015).

Table 4. Comparison between deep learning and reference models for flood inundation.

\begin{tabular}{cccccl}
\hline \multirow{2}{*}{$\begin{array}{c}\text { Case study } \\
\text { size }\left[\mathrm{km}^{2}\right]\end{array}$} & Model & Metric & Model & Metric & Reference \\
\hline 3600 & MLP+PSO & Accuracy $=81.6 \%$ & PSO & Accuracy $=79.7 \%$ & Li et al. (2016a) \\
5625 & MLP+GA & Accuracy $=81 \%$ & GA & Accuracy $=79.3 \%$ & Li et al. (2015) \\
0.01 & CNN & Accuracy $=95.5 \%$ & SVM & Accuracy $=87.4 \%$ & Gebrehiwot et al. (2019) \\
0.9 & CNN & IoU $=84 \%$ & SVM+RBF & IoU $=83 \%$ & Nogueira et al. (2017) \\
59.3 & CNN & Accuracy $=93.7 \%$ & SVM & Accuracy $=92.1 \%$ & Peng et al. (2019) \\
25000 & CNN & Accuracy $=97 \%$ & ATM & Accuracy $=91 \%$ & Nemni et al. (2020) \\
31450 & CNN & Accuracy $=65.3 \%$ & SVM & Accuracy $=55.3 \%$ & Sarker et al. (2019) \\
\hline
\end{tabular}

$\mathrm{PSO}=$ particle swarm optimization; $\mathrm{GA}=$ genetic algorithm; $\mathrm{SVM}=$ support vector machine $; \mathrm{RBF}=$ radial basis function; ATM = automatic threshold model

\subsection{Deep Learning for flood hazard}

Flood hazard predicts the depth, velocity, and extent of floods. This application produces maps which evaluate for a certain event its maximum inundation (e.g., Guo et al., 2020; Berkhahn et al., 2019; Löwe et al., 2021) or how it evolves in time (e.g., 
Lin et al., 2020a; Zhou et al., 2021). While most studies consider the probability of different events, using return periods (e.g., Kabir et al., 2020; Guo et al., 2020), there are few papers which determine the water depth map for a single event (e.g., Hu et al., 2019; Chang et al., 2010). However, no papers were identified to predict the flow velocities. Since the simulation results are taken as ground-truth data to train the deep learning models, deep learning models for flood hazard mapping are used as surrogate models in place of the standard numerical models.

The most studied types of floods are river and urban floods. As regards the spatial scale, the models are carried out at local and regional scales. This is probably due to the computational burden of performing several simulations at larger scales to train the deep learning model.

\subsubsection{DL architecture}

The deep learning models are mainly based on MLPs and RNNs. In particular, RNNs are applied when a spatial-temporal estimation of the water depths is performed. CNNs were initially discarded but are used more in recent years (Guo et al., 2020; Löwe et al., 2021; Kabir et al., 2020).

\subsubsection{Input and output data}

The inputs are hyetographs, which represent the rainfall precipitation or intensity in time (e.g., Berkhahn et al., 2019; Kao et al., 2021; Guo et al., 2020) or hydrographs, which represent the discharge in time (e.g., Chu et al., 2020; Zhou et al., 2021; Lin et al., 2020a). Other inputs such as the DEM and the roughness coefficient, also used for numerical models, are sometimes considered as additional inputs (e.g., Guo et al., 2020; Chang et al., 2010; Huang et al., 2021b). However, no analysis has been performed to evaluate whether their addition is beneficial.

The output is a water depth map. For the datasets, it is obtained via numerical models based on the 2D shallow water equations. 1D, 1D-2D, and 3D models are also used (Kao et al., 2021; Chang et al., 2010; Hu et al., 2019). The main reason why numerical models are used is to simulate events that have never occurred or have never been observed, such as floods with high return periods. Even though observed data were not employed, they could be used in future research to corroborate the transferability of such methods. When training only on the numerical models' predictions, the deep learning models' results are limited in accuracy by the numerical models' one, i.e., if the numerical model does not represent reality so will the DL model. Thus, when the model is deployed on real data, there may also be some generalizability issues caused by the difference between the training and testing data. The inclusion of real measured data may thus also improve the accuracy with respect to numerical models.

\subsubsection{Performance assessment}

In flood hazard regression metrics are used to evaluate the water depth while classification metrics are used to evaluate the flood extent, as done for flood inundation (Fig. 6). While for flood susceptibility and inundation DL models were used to improve the performances, in flood hazard their main focus is to improve the speed, while still maintaining reasonably low errors with 
respect to the numerical predictions, as highlighted in Table 5. We define the speed-up as the ratio between the computational times of the numerical model and the DL model.

Table 5. Comparison between deep learning and numerical models for flood hazard.

\begin{tabular}{cccccl}
\hline $\begin{array}{c}\text { Case study } \\
\text { size }\left[\mathrm{km}^{2}\right]\end{array}$ & $\begin{array}{c}\text { Deep learning } \\
\text { Model }\end{array}$ & $\begin{array}{c}\text { Numerical } \\
\text { Model }\end{array}$ & $\begin{array}{c}\text { Comparison } \\
\text { measure }\end{array}$ & Speed-up & Reference \\
\hline 0.6 & MLP & 2D & RMSE $=0.0013 \mathrm{~m}$ & $1000 \mathrm{x}$ & Berkhahn et al. (2019) \\
7.7 & MLP & 2D & RMSE $=0.16 \mathrm{~m}$ & $100 \mathrm{x}$ & Chu et al. (2020) \\
31 & MLP & 1D-2D & MAE $=0.06 \mathrm{~m}$ & $1000 \mathrm{x}$ & Chang et al. (2010) \\
10.5 & $\mathrm{CNN}$ & 2D & $\begin{array}{c}\mathrm{MAE}<1 \mathrm{~m} \\
\text { for } 93 \% \text { of domain }\end{array}$ & $2000 \mathrm{x}$ & Guo et al. (2020) \\
14.5 & $\mathrm{CNN}$ & 2D & RMSE $=0.11 \mathrm{~m}$ & $38 \mathrm{x}$ & Kabir et al. (2020) \\
18.5 & RNN & 3D & RMSE $=0.01 \mathrm{~m}$ & $1500 \mathrm{x}$ & Hu et al. (2019) \\
1479 & RNN & 2D & RMSE $=0.056 \mathrm{~m}$ & $21 \mathrm{x}$ & Zhou et al. (2021) \\
\hline
\end{tabular}

\section{Knowledge gaps}

We identified knowledge gaps regarding the applications in flood management, usability, modeling limitations, and data availability. Some other minor gaps were shown in the previous section. Based on these gaps, future research directions are proposed in Section 5.

\subsection{Flood applications and usability}

Deep learning has proven useful for assessing flood-prone areas from the location of past events, identifying flooded areas from remote sensing images, and working as a surrogate model for numerical simulations. However, other applications within this field still could benefit from deep learning models. In particular, we address two flood management applications, flood risk and real-time flood warning. We also define two desired types of maps, flood arrival time maps and probabilistic hazard maps. Then we discuss dam and dike breach flood events.

Flood risk combines the probability that a certain event occurs with the associated consequences, such as economic impacts or loss of life. The expected annual loss is a common measure obtained from flood risk assessment and depends on (i) flood hazard, given by event-specific flood characteristics, such as water depth and flow velocity, (ii) exposure, related to the elements at risk, such as buildings and critical infrastructures, and (iii) vulnerability, i.e., the inability of a system to withstand the effects of the event, given, for example, by intensity-damage curves. Flood risk maps are obtained by combining flood hazard maps with damage models. Other approaches are based on MCDA, since the exact flood magnitude and damage are uncertain (de Brito and Evers, 2016). This is done by incorporating various factors that determine flood risk, such as hazard, 
the performance of defenses, topography, and exposure. However, MCDA is based on expert knowledge and is thus subjective. DL models solve this issue and can also yield a higher accuracy, as shown for flood susceptibility mapping. Thus, DL-based approaches could provide alternative methods for assessing flood risk. In addition to the inputs used for flood susceptibility, such as elevation and land use, flood risk mapping may require also other inputs such as population density, spatial estimates of economic value, and building types. Up to now, only Chen et al. (2021) combined DL and flood risk assessment. They showed that ML and DL approaches can estimate flood risk at regional scale, but do not compare their results against other methods, such as MCDA. One drawback of their approach is that the resulting maps were qualitative, while quantitative results should be preferable for risk assessment.

Real-time flood warning is another application that has not been widely addressed. This is needed by local authorities to inform when and where a flood may occur. While several papers mention real-time prediction, most can be used only after the event has occurred, since they require as input the complete hyetograph or hydrograph of the event. There are a few examples based on RNNs which could forecast floods in near real-time using sensors (Kao et al., 2021) and rainfall distribution (Dong et al., 2021). However, few situations are covered and, thus, more research should focus on filling this gap. An alternative method is to predict the rainfall in real-time and then retrieve the corresponding water depth map by using a similarity measure on a large dataset of previous simulations (Chang et al., 2020). However, such a solution may be challenging because of the large storage requirements. Using DL for surrogate modeling instead showed substantial speed improvements, thus allowing for real-time simulations and forecasts. Similar achievements have already been obtained for rainfall now-casting, where the deep learning models can accurately forecast the near-future rainfalls (e.g., Shi et al., 2015; Ravuri et al., 2021).

Arrival time maps estimate the time employed by a flood to reach a certain water depth threshold. They can encode both spatial and temporal information in the same map. So, for a practitioner, they carry at one place detailed information not only on where to intervene but also when to execute mitigation measures. Despite these promises, they have been seldom used in flood management; consequently, they have also not been exploited with DL methods. Using DL for arrival map estimation may be a promising direction to identify critical infrastructure and set up corresponding evacuation plans in real-time. This is because DL has shown potential for surrogate modeling (see Table 5) and because arrival maps can be obtained from flood hazard maps taken over different time intervals of a flood event. This application may be particularly important for exceptional flood events, such as dike breaches and dam breaks, where little forecast can be made until a failure initiates (Yakti et al., 2018).

Probabilistic hazard mapping captures the model uncertainty related to its inputs and outputs. As pointed out by Di Baldassarre et al. (2010), uncertainties can result in deterministic maps which are only spuriously accurate. But, probabilistic maps can account for the uncertainties by assigning a probability of flooding to each domain element. This analysis is generally carried out with probabilistic methods such as Monte Carlo simulations (e.g., Papaioannou et al., 2017). However, since they require a vast amount of simulations, only simpler numerical models are used. DL models could be used as surrogates to speed up computation and improve the accuracy of the simpler models. Nonetheless, brute-force simulations, such as Monte Carlo, require up to hundreds of thousands of simulations to obtain a satisfactory measure of the uncertainty (Liu, 2017). Thus, we need models that can intrinsically work with probabilistic input distributions of parameters. 
Dam and dike breach floods concern a relevant category of flood events that have not been yet approached with deep learning models. The motivation is probably related to the rarity of such events and the complexity of the phenomena. Their catastrophic and unexpected effects make their modeling necessary in several situations. However, current DL models seem to be unable to do so. Moreover, the effect of flood defenses' failure is often disregarded also because the location and modality of possible failures are uncertain. A common way to include the failure of structures is to investigate all possible combinations of locations and boundary conditions, but it can be constrictive both for time and storage capacities. Probabilistic hazard mapping may be a relevant application to include the uncertainty in the failure probability of the flood defense (Domeneghetti et al., 2013).

\subsection{Modeling limitations}

Current applications of deep learning for flood mapping have encountered some limitations. The two main issues are related to their generalization ability across different case studies and the modeling of complex interactions.

Except for flood inundation mapping, only a few papers tested the model on different areas rather than the one trained on. This is particularly useful because training requires data, model development, and time. However, this transference between areas is challenging for DL models because of the difference in input and output data. Löwe et al. (2021) generate flood hazard maps also for areas that were not considered during training. These areas were within the same study region, thus there was little variability of inputs and outputs with respect to the training set. Zhao et al. (2021b) instead pre-trained a model for flood susceptibility on an urban area and then used it for another similar area. They showed that pre-training improves predictions with respect to a model trained from scratch, both in cases of low and high data availability. A DL model which cannot generalize to new areas has to be trained every time for a new study case. Thus, it may have limited advantages over a hydraulic model, since it requires more effort, data, and time. Instead, a general DL model which can generalize to new areas could emphasize the advantages over numerical models. This concept was experimented also for rainfall-runoff modeling where DL models outperformed state-of-the-art alternatives in the prediction of ungauged basins in new study areas (Kratzert et al., 2019b).

Complex interactions with the natural and built environment, such as dikes or buildings, are difficult to include in deep learning models. Kabir et al. (2020) showed that flood defenses can be included if they are present in the simulations used for training and testing. However, no solution presented so far can directly include new flood defenses in it. While buildings may be included in the DEMs, bridges and other hydraulic structures that influence the behavior of the floods may be harder to include, due to their strong influence on the flow path.

\subsection{Data availability}

Deep learning models usually require large quantities of data to achieve good performances. While simulations can provide potentially limitless data, observed data are scarce and depend on the study area. Simulations may also encounter instability issues depending on the numerical schemes and study area. Remote sensing has provided large quantities of data since its vast development in the past decades, but satellite data is still limited by its frequency of observations and dependency on favorable meteorological conditions. Also, UAVs cannot cover wide areas at once. Precipitation and water depth data are available only 
in a few locations where the measuring stations are present. Thus, new data sources are needed to overcome these limitations. Several papers already employ camera images to detect floods and measure the associated water depth (e.g., Feng et al., 2020; Jafari et al., 2021). The acquisition of photos in a consistent way could help in providing data in previously hard to obtain areas, such as urban environments. Social media information can also be used to identify flooded areas (e.g., Rossi et al., 2018). However, the heterogeneity of the sources of these data needs to be carefully taken into account when deploying a DL model.

\section{Future research directions}

The present review shows that flood practitioners still need to be up to date with the latest and most successful deep learning models. We suggest that the outstanding identified issues can be approached by resorting to Deep Learning state-of-the-art advancements to our field. As such, we propose future research directions to transfer this knowledge and address the aboveidentified gaps.

\subsection{Mesh-based Deep Learning}
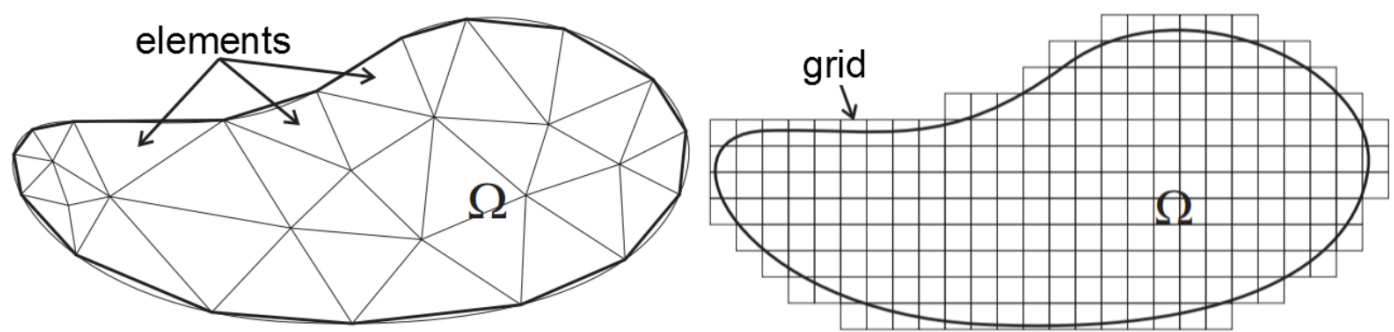

Figure 7. The irregular geometrical structure of the mesh allows capturing information in a more efficient way than regular grids by following the properties of the underlying system (picture taken from (Ferreira et al., 2015)).

Current deep learning models lack generalization across different situations, meaning that they can work exclusively for a specific purpose or area. They also cannot represent complex interactions with the natural and built environment. Both issues depend on the regular grids used in the reviewed papers, which are unable to follow the geometric properties of irregular inputs as illustrated in Fig. 7. Hence, the model cannot exploit many data patterns, ultimately limiting its generalizability and, for the same motivation, being unable to account for the irregular geometrical structures. Unstructured meshes can solve this problem by discretizing the domain (Mavriplis, 1997). A mesh is a structure composed of a collection of nodes, edges, and faces used to discretize a continuous domain. Meshes are commonly used for numerical simulations in many physical systems (e.g., Ferraro et al., 2020; Bomers et al., 2019). Their flexible definition allows to increase the resolution where needed and coarsen it otherwise, ultimately decreasing the computational time and improving efficiency (Candy, 2017). However, standard DL models, such as CNNs, cannot be applied on meshes. There are currently several lines of work which, instead, can use 
meshes as a learning framework. They are here referred to as mesh-based neural networks. Two highly promising mesh-based approaches for flood applications are geometric deep learning and neural operators.

Geometric deep learning extends the concepts of deep learning to non-Euclidean domains, such as graphs and manifolds (Bronstein et al., 2017). This is possible by preserving symmetries with respect to more transformations, such as permutations and rotations. These additional inductive biases decrease the required data and allow working with meshes. For example, permutation equivariance means that operations are independent of the ordering of the nodes and elements of the mesh. Hence, the model can recognize different geometric patterns without any necessity of data augmentation, i.e., manually increasing the data by applying transformations to it. Thus, similar properties in the data can be inherently recognized and exploited. Among the several geometric deep learning models which can work with meshes, graph neural networks (GNN) are the most developed ones. Graphs are structures defined by a set of nodes and edges and can be considered as the underlying skeleton of a mesh. GNNs allow to model data on graphs by considering how its elements are connected (Wu et al., 2021; Gama et al., 2020). GNNs take as input the information encoded in the nodes, in the edges, and in the graph structure, and then process it with neural networks, in a similar way as done by CNNs and RNNs with grid elements and sequential data, respectively. For example, nodes can carry information on the elevation of a point or its boundary conditions, while edges may encode the spatial distance between nodes. Several variations of GNNs exist that give more importance to certain parts of the data by weighting information from different neighbours (Wu et al., 2021; Isufi et al., 2021). There also exist promising works which simulate fluid dynamics with mesh-based GNNs. Pfaff et al. (2021) used a GNN-based model to perform simulations for different physical systems, showing increased performance and stability with respect to CNN models on the same domains. For each node, they used information related to their position, type of node, and flow conditions. Lino et al. (2021) used a similar model to simulate advection and incompressible flows. The model also considers different resolutions to improve the information propagation at larger scales. However, GNNs consider only pairwise geometrical properties as connections between nodes, thus neglecting the mesh structure. Recent developments focused on extend the GNN framework to include it. Mesh convolutional neural networks adapt GNNs to include a representation of the local geometry, which preserves the angles between edges (De Haan et al., 2020; Zhou et al., 2020). Simplicial neural networks (Yang et al., 2021; Ebli et al., 2020) and cell complex neural networks (Bodnar et al., 2021; Hajij et al., 2020), instead, generalize GNNs to higher-order structures. They can consider as well information on triangular and polyhedral elements, which can represent, for example, a flooded area or a volume. This inclusion of the mesh properties in such approaches may further enhance the power of GNNs. Even though they are still in their infancy, their potential for learning on meshes could reveal to be useful also for flood modelling in future research.

While promising, the aforementioned approaches ignore any underlying physical laws present in flood modelling and let the model figure them out. But these physical laws provide additional inductive biases, hence we could include them in modeling to enhance the performance. Physics-based DL and neural operators are approaches that account for them. Physics-informed neural networks (PINN) employ physical laws to constraint the model solution (Raissi et al., 2019). The idea is to parameterize a partial differential equations (PDE) solution with a neural network, while keeping the same physical formulation. Then, each partial derivative in the equations is determined via automatic differentiation. Many works have shown the capabilities 
of PINNs to follow the underlying PDEs in fluid dynamics (e.g., Mao et al., 2020; Yang et al., 2019b). This is relevant also in flood modelling where PDEs such as shallow water equations or the Navier-Stokes equations are employed. However, they model only one specific instance of the PDE, not the solution operator (Kovachki et al., 2021). Neural operators, instead, can learn mappings between function spaces, i.e., they learn a whole family of equations (Lu et al., 2019). In other words, they can approximate any differential operators. Moreover, since neural operators learn a mapping between infinite-dimensional spaces, they are invariant with respect to the chosen discretization. Thus, their solution is transferable to any mesh resolution. Among the proposed models, Fourier neural operators (FNO) have currently achieved the best results Li et al. (2021). The basic idea is to extract features from the input function, process them in the function space, and, finally, map them to the output function. In FNOs, the function space is given by the Fourier space, which allows to use fast Fourier transforms. Results show that FNOs improve the speed of several PDEs by up to three orders of magnitude. Consequently, they could be used in flood management to overcome computational speed limitations while preserving the underlying physics, allowing also for more reliable real-time flood warning. Thanks to the inductive biases given by the physical laws, neural operators also require less data.

\subsection{Probabilistic Deep Learning}

Uncertainties in floods are often determined via probabilistic hazard mapping. These maps show the inundation depths and extents together with their confidence intervals and are traditionally obtained with Monte-Carlo simulations (e.g., Domeneghetti et al., 2013). To avoid brute-force simulations and provide uncertainty guarantees, certain deep learning models can consider uncertainty in the model inputs. An example of these models is deep Gaussian processes (DGP). DGP are models composed by the stacking of Gaussian processes (GP), in a similar fashion done in neural networks (Damianou and Lawrence, 2013). A GP is a collection of random variables whose joint distribution is a Gaussian (Rasmussen, 2003). They benefit from the properties of normal distributions and thus their output can be obtained analytically. The advantage of DGPs over GPs is that they can better extract patterns in data thanks to their increased complexity. DGPs can determine the distribution of the output and could, therefore, be used in probabilistic hazard modeling to determine the range of variation of the predicted flood hazard map. No example of DGPs used for flood mapping exists yet. However, GPs have been used for the statistical estimation of the correlation between flooding and sea-level rise (Vandenberg-Rodes et al., 2016).

\subsection{Data scarcity}

Even though remote sensing and measuring stations provide noticeable amounts of data, several parts of the world still lack enough data to deploy deep learning models.

A possibility is to generate artificial data, especially in areas or situations where it is hard to obtain. This can be done using generative adversarial networks (GAN), which create new data from a given dataset (Goodfellow et al., 2014). GANs are composed of two neural networks, named generator and discriminator, whose purpose is, respectively, to generate new data and to detect if a given data is real or fake. A trained GAN can produce new fake but plausible data. Lütjens et al. (2020) and Lütjens et al. (2021) use GANs to generate realistic post-flood inundation maps in coastal and arctic areas when clouds block satellites from taking real-time pictures. Given a set of pre-flood maps and flood masks, i.e., the flood extent detected 
by radar, their models can provide plausible post-flood maps for emergency purposes. GANs can be used as well to generate predictions, such as meteorological forecasts (Ravuri et al., 2021). They have also been used to create realistic scenarios of natural disasters, such as floods, for projected climate change variations (Schmidt et al., 2019). Moreover, GANs could be used to generate a plausible urban drainage system or topography for cities that do not have any construction plan for their sewers and in areas where only low-resolution data is available, as was done in similar fields (e.g., Fang et al., 2020b).

However, GANs are difficult to train (Goodfellow, 2016). Variational autoencoders (VAE) are another type of generative model, which can overcome this issue. Differently from standard autoencoders, VAEs model the latent space with probability distributions that aim to ensure good generative properties to the model (Kingma and Welling, 2013). Once the model is trained, new synthetic data can be generated by taking new samples from the latent distributions. Nonetheless, because of the model's definition, the predictions are less precise than GANs. As such, VAEs and GANs offer a trade-off between the reality of the prediction and the availability of training data.

Another way to deal with data scarcity is to use transfer learning. This refers to the model trained on a dataset and deployed on different ones. This approach was used by Zhao et al. (2021b) for flood susceptibility mapping showing that a pre-trained model improves the mapping's accuracy for data-scarce areas. Muñoz et al. (2021) also used it and demonstrated that an inundation model trained on a local scale can be applied to broader scales. Thus, transfer learning carries great potential to bridge the data scarcity gap also in other scenarios.

\section{Conclusions}

This paper presented a review of current applications of deep learning models for flood mapping. The search criteria focused on DL applications in flood mapping since 2010, leading to a total of 45 reviewed papers. Our main conclusions can be summarized as follows:

- Flood susceptibility, inundation, and hazard mapping were investigated using deep learning models. In flood susceptibility, deep learning models consider several inputs, the most important being slope, land use, aspect, terrain curvature, and distance from the rivers. The main deep learning model used were MLPs, often in combination with other statistical techniques. Flood inundation considers as the main data images of floods, mostly taken via satellite. The main deep learning models were CNNs. Flood hazard maps estimate the water depth in a study area by using deep learning as a surrogate model for numerical simulations. For this application, there are no deep learning model preferences. Regardless of the application, results show that deep learning solutions outperform traditional approaches as well as other ML techniques.

- MLPs and CNNs were the most common type of deep learning model considered in flood mapping, while RNNs were less used. To overcome their lack of inductive biases and achieve good accuracy, MLPs are often coupled with other statistical techniques. On the other hand, thanks to their spatial and temporal inductive biases, standalone CNNs and RNNs were found to regularly outperform other models. DL models used for surrogate modeling provide significant speed-up (up to three orders of magnitude) while maintaining sufficient accuracy. 
- Most papers dealt with river and urban floods, while only a few works described applications for flash and coastal floods. Case studies were mainly addressed at local or regional scales, arguably due to the availability of high-resolution data. Conversely, the community should further investigate the suitability of DL models for flood applications at larger scales. Concerning the development data, we found that models producing susceptibility and inundation maps rely on the availability of real flood observations. Finally, training DL-based surrogate models for hazard mapping requires target data from numerical simulations.

This review outlined several knowledge gaps, which can be addressed via deep learning to improve the state of the art of flood mapping. To solve these gaps we proposed possible solutions based on recent advances in fundamental machine learning research:

- Flood risk could be addressed in a similar way as for flood susceptibility by using physical and economical characteristics to obtain a risk map. Flood arrival time maps can provide both spatial and temporal information of a flood event and may be obtained similarly as for flood hazard maps.

- Current deep learning models cannot generalize across different case studies and regions, meaning that a new model has to be created each time. Moreover, current models cannot account for the complex interactions with the natural and built environment. A solution to these problems is to use novel DL architectures that include meshes as learning frameworks. Mesh-based neural networks, such as graph neural networks and neural operators, can consider arbitrarily shaped domains and thus provide the required flexibility to generalize across case studies and model the effects of complex interactions.

- Real-time flood warning can be modeled via fast surrogate models based on deep learning. Fourier neural operators provide a reliable framework since they consider the underlying physical equations. Probabilistic hazard mapping can take advantage of deep Gaussian processes to determine the uncertainties associated with the model and the inputs. Dam and dike breach floods can be modeled using geometric deep learning models, thanks to their capability to deal with complex situations.

- DL necessitates large quantities of data which are difficult to collect in several areas of the world. Generative models such as GANs and VAEs can be employed to produce synthetic data for such data-scarce regions, based on training data collected elsewhere. Furthermore, the community should better investigate the potential of transfer learning and the adoption of pre-trained models, which may substantially reduce data requirements.

We expect deep learning to be a promising tool to improve and speed up flood mapping. Nonetheless, deep learning models are black-box models, meaning that the underlying operations are unknown. Thus, their deployment in real emergencies has to be taken cautiously. As deep learning for flood mapping is still novel, we advise its use in critical situations to be always validated by traditional models and expert knowledge, until robust and corroborated models are available. The above concern highlights the main challenge DL models for flood management need to face. However, DL models are still in their infancy and 
https://doi.org/10.5194/hess-2021-614

Preprint. Discussion started: 13 December 2021

(c) Author(s) 2021. CC BY 4.0 License.

carry the large potential to aid researchers for many applications, especially where traditional models cannot provide sufficient accuracy or speed. In particular, deep learning-based flood mapping approaches could provide an added value for regions with limited data or limited resources to invest in setting up time-consuming hydraulic models.

\section{Appendix A: Flood susceptibility inputs}

Figure 1a shows the complete list of the inputs used in the flood susceptibility models. Inputs which were considered only once were discarded from this graph.

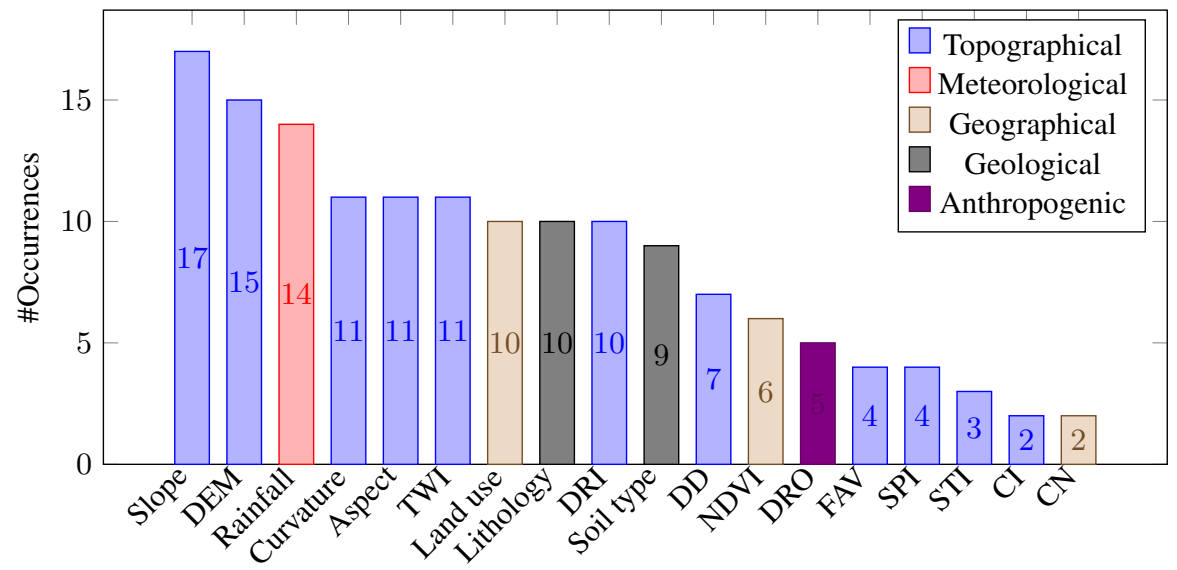

Figure A1. Distribution of the inputs for flood susceptibility for the 17 reviewed papers. The inputs are categorized in topographical, meteorological, geographical, geological, and anthropogenic factors. Inputs which were considered only once were discarded from this graph.

Abbreviations: $\mathrm{CI}=$ Convergence Index; $\mathrm{CN}=$ Curve Number; DD = Drainage Density; DEM = Digital Elevation Model; DRI = Distance from Rivers; DRO = Distance from Roads; FAV = Flow Accumulation Value; NDVI = Normalized Difference Vegetation Index; SPI = Stream Power Index; STI = Sediment Transport Index; TWI = Topographic Wetness Index

\section{Appendix B: Comparison metrics}




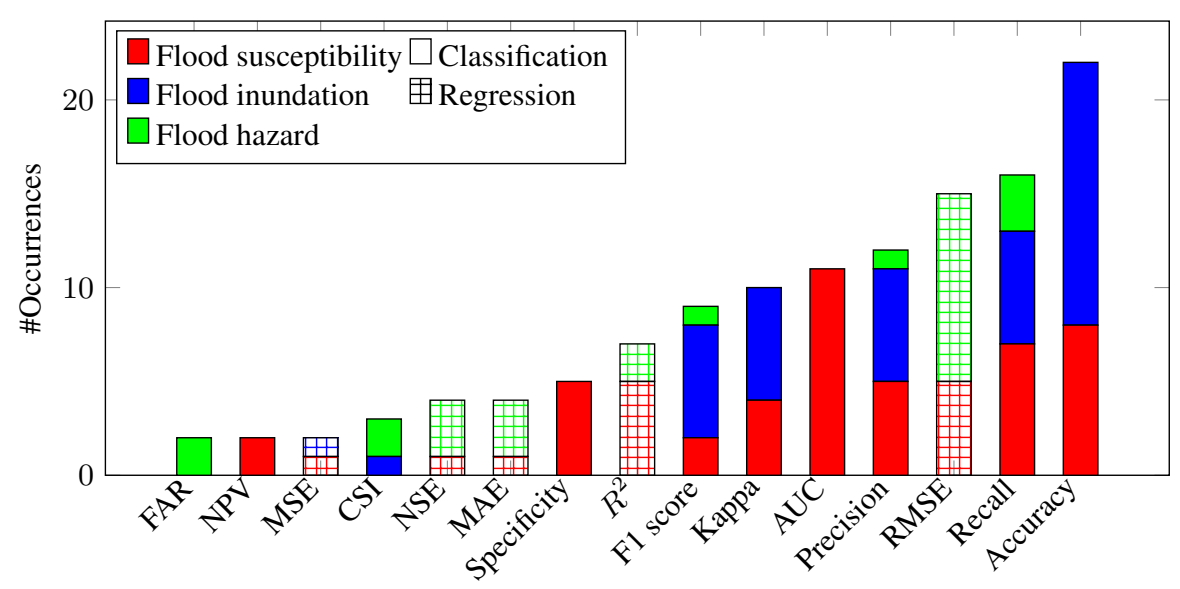

Figure B1. Distribution of the comparison metrics in the reviewed papers per type of application.

Abbreviations: AUC $=$ Area Under the ROC Curve; CSI = Critical Success Index; FAR = False Alarm Ratio; NPV $=$ Negative Predictive Value; RMSE = Root Mean Squared Error; $R^{2}=$ Coefficient of determination; MAE = Mean Average Error; NSE = Nash-Suitcliffe Efficiency; MSE = Mean Squared Error

\section{Appendix C: Reviewed papers}

Table C1: A brief description of the reviewed papers, following the same ordering as in Table 2.

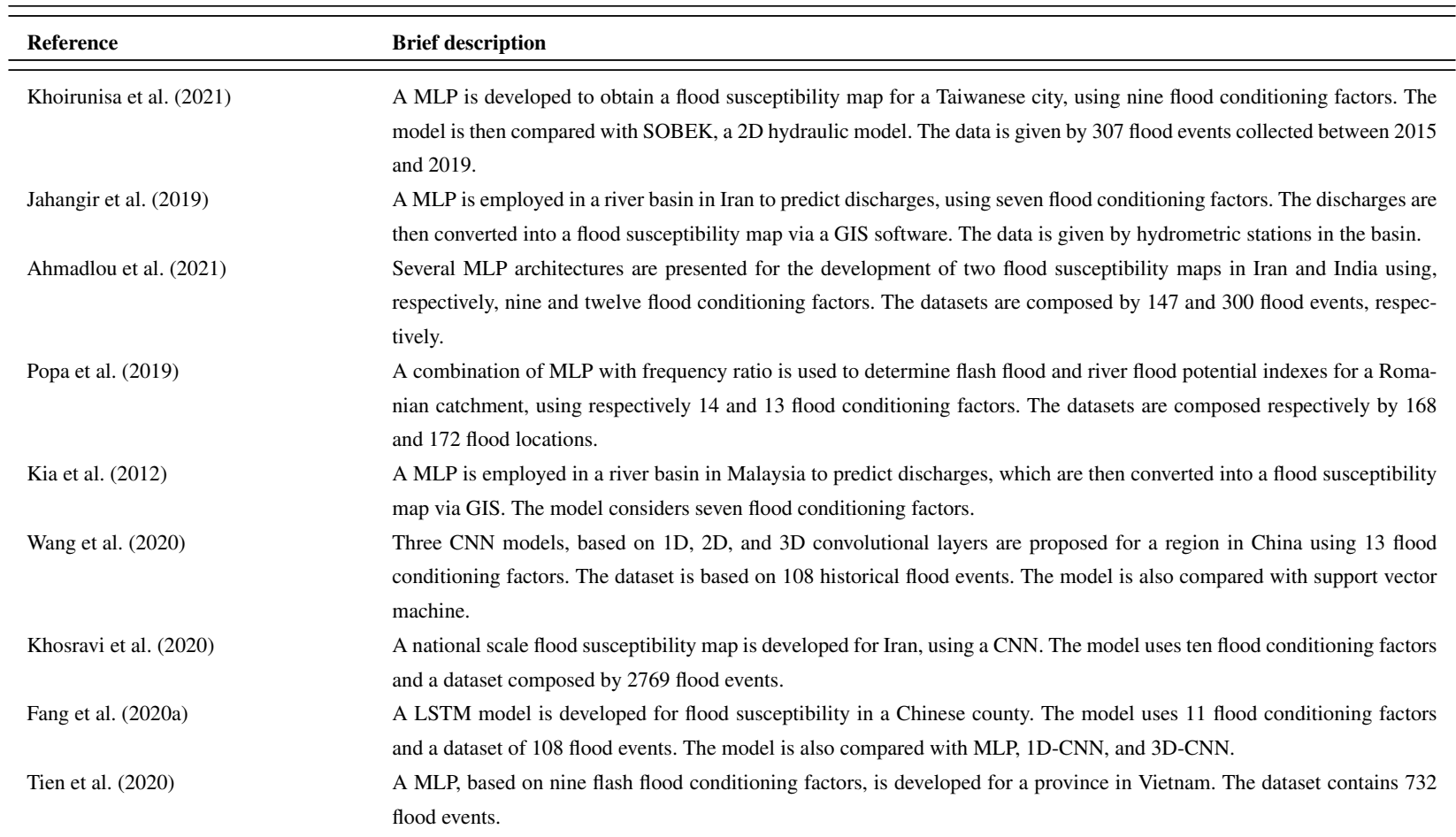


Ngo et al. (2018)

Costache et al. (2020)

Kourgialas and Karatzas (2017)

Panahi et al. (2021)

Darabi et al. (2021)

Kalantar et al. (2021)

Zhao et al. (2020b)

Zhao et al. (2021b)

A combination of MLP with firefly algorithm optimization technique is proposed for flash flood susceptibility in a Vietnamese region. The model considers 12 flood conditioning factors and a dataset of 654 flooded areas

Two MLP models, combined with analytical hierarchy process and frequency ratio, respectively, are developed for flash flood susceptibility mapping in a catchment in Romania. The model considers ten flood conditioning factors and 178 flooded locations.

A national-scale flash flood susceptibility map is obtained with a MLP for Greece. The model considers seven flood conditioning factors and a dataset of 600 flood events.

Two models, CNN and RNN, are developed for flash flood susceptibility in a province in Iran. The model considers nine flood conditioning factors and a dataset of 143 flood events.

A MLP ensemble model is used for urban flood susceptibility mapping in a Iranian city. The model uses six flood conditioning factors and a dataset of 118 flooded locations.

Three MLP models, one of which optimized with particle swarm optimization, are developed for flood susceptibility mapping in a urban catchment in Australia. The model considers 13 flood conditioning factors and 128 flooded locations. Two CNN models are used to assess urban flood susceptibility for a Chiese catchment. The model considers nine flood conditioning factors and 202 flooded locations. The model is also compared with support vector machine and random forest.

A pre-trained CNN model, taken from Zhao et al. (2020b), is deployed in two urban areas, with data-rich and data-scarce scenarios.

Li et al. (2015)

A MLP combined with genetic algorithm is used for super-resolution mapping of wetland inundation. Images are taken from Landsat remote sensing for two areas, in China and Australia.

Li et al. (2016a)

Nogueira et al. (2017)

Gebrehiwot et al. (2019)

Ichim and Popescu (2020)

Hou et al. (2021)

A MLP optimized with particle swarm optimization is used for super-resolution mapping. Images are taken from Landsat remote sensing for two areas, in China and Australia.

Satellite image patches, collected from eight flooding events, are classified in flooded and non flooded areas using a CNN. A set of images taken from unmanned aerial vehicles is used for flood inundation mapping using a CNN. The dataset is composed by three study areas in North Carolina, USA, with a total of 100 images.

A combination of five different CNN based models is used for flood inundation mapping. A set of 2000 images derived from UAVs in a Romanian rural region is classified into flooded, vegetation and non-flooded areas.

A flood propagation experiment in a small scale laboratory is used to develop a CNN model for inundation mapping. The dataset is composed by a sequence of 1930 images detected from cameras.

Hashemi-Beni and Gebrehiwot (2021)

Kang et al. (2018)

Sarker et al. (2019)

Nemni et al. (2020)

Amini (2010)

Li et al. (2016b)

Peng et al. (2019)

Dong et al. (2021)

Liu et al. (2019)

Muñoz et al. (2021)

Chu et al. (2020)

Lin et al. (2020b)

A encoder-decoder CNN is developed for flood inundation mapping. The study area is the same as Gebrehiwot et al. (2019).

A CNN model is developed for flood detection from satellite images for three study areas in China.

A CNN model is trained to predict flood extent from Landsat satellite images across Australia.

A CNN model is trained to predict flood extent from Sentinel-1 SAR imagery. The UNOSET flood dataset, which covers flood events from Africa and East Asia, is used for the model development.

High-resolution images are classified into five categories with a MLP. The study area is located in a Iranian city.

A MLP is developed for super-resolution mapping. Images are taken from Landsat remote sensing for two areas, in China and Australia.

A CNN which considers pre- and post-flood satellite images is used for urban flood detection. The study area is in Texas, USA, and considers two hurricane events.

A combination of 1D-CNN and GRU is used for prediction of flood inundation in urban area in Texas, USA. The dataset is composed by a channel sensor network. Given information on the temporal evolution of water depth and precipitation, for each sensor, the model predicts which nodes will be flooded.

A CNN which considers pre- and post-flood SAR images is used for coastal flood detection. The study area is in Texas, USA, and considers six pairs of satellite images for a hurricane event.

A CNN model is developed for compound flood mapping for the Atlantic coast of USA. The model takes as input Landsat and SAR satellite data, along with a DEM of the study areas, and combines them with data fusion. The model distinguishes then between several categories, such as flooded areas and vegetation.

A MLP is used to estimate the water depth for a river reach in Australia. The dataset is generated using a 2D hydrodynamic model, TUFLOW, and considers ten flood events.

A MLP is used to estimate the water depth and extent for a river reach in a German city. The dataset is generated using a 2D hydrodynamic model, HEC-RAS, and considers 180 flood events. 
https://doi.org/10.5194/hess-2021-614

Preprint. Discussion started: 13 December 2021

(c) Author(s) 2021. CC BY 4.0 License.

Lin et al. (2020a)

Huang et al. (2021a)

Kabir et al. (2020)

Guo et al. (2020)

Zhou et al. (2021)

Kao et al. (2021)

Chang et al. (2010)

Berkhahn et al. (2019)

Löwe et al. (2021)

Hu et al. (2019)
A MLP is used to forecast water depth and flood extent for a river reach in a German city. The dataset is the same as in Lin et al. (2020b).

Two models, MLP and RNN, are developed for water depth estimation for an American river. Before being given as input to the models, the data is clustered based on slope, drainage area and hydrologic length. The dataset is generated using a 2D hydrodynamic model and considers 20 flood events.

A 1D-CNN model is used to predict a flood hazard map, from a discharge hydrograph, for a river reach in England. The dataset is generated using a 2D hydrodynamic model, LISFLOOD-FP, and considers 10 flood events. The model is also compared with support vector regression.

A encoder-decoder CNN is used for flood mapping in three catchments, two in Switzerland and one in Portugal. The dataset is generated using a cellular-automata flood model, CADDIES, and considers 18 flood events.

A LSTM model is used to predict the temporal evolution of water depth in few representative locations along an Australian river. The water depths are then interpolated to obtain a flood hazard map. The dataset is generated using a 2D hydrodynamic model, TUFLOW, and considers 74 flood events.

A stacked encoder decoder LSTM is used to determine flood hazard maps in time from precipitation. The study area is located in Taiwan. The dataset is generated using a storm water management model, HEC-1, plus a $2 \mathrm{~d}$ hydrodynamic model and considers 24 flood events.

A MLP is developed to forecast one hour ahead flood hazard maps in a Taiwanese county. The data is also pre-processed by clustering. The dataset is generated using a storm water management model, HEC-1, plus a $2 \mathrm{~d}$ hydrodynamic model and considers 120 flood events.

A MLP is used to predict maximum water levels for two urban areas. The dataset is generated using a coupled sewer-surface model, HE 2D, and considers 64 flood events.

A encoder-decoder CNN is used for flood mapping in a urban area in Denmark. The dataset is generated using a 2D Hydrodynamic model, MIKE 21, and considers 53 flood events.

A LSTM model is developed to simulate a tsunami in Japan. The model is trained in a lower dimensional space to reduce the problem's complexity. The dataset is obtained using a 3D hydrodynamic model, Fluidity, and consists of 100 snapshots of a modelled tsunami event.

Author contributions. All authors contributed in conceptualizing the paper and its contents. RB and RT developed the structure of the paper. RB wrote the paper, produced all figures and tables, and formatted the article. RT, EI and SNJ reviewed, revised, and supervised the progress of paper.

Competing interests. No competing interests are present.

Acknowledgements. This work is supported by the TU Delft AI Labs programme. 


\section{References}

Abdullah, M. F., Siraj, S., and Hodgett, R. E.: An Overview of Multi-Criteria Decision Analysis (MCDA) Application in Managing WaterRelated Disaster Events: Analyzing 20 Years of Literature for Flood and Drought Events, Water, 13, https://doi.org/10.3390/w13101358, 2021.

Ahmadlou, M., Al-Fugara, A., Al-Shabeeb, A., Arora, A., Al-Adamat, R., Pham, Q., Al-Ansari, N., Linh, N., and Sajedi, H.: Flood susceptibility mapping and assessment using a novel deep learning model combining multilayer perceptron and autoencoder neural networks, Journal of Flood Risk Management, 14, 2021.

Alcrudo, F. and Garcia-Navarro, P.: A high-resolution Godunov-type scheme in finite volumes for the 2D shallow-water equations, International Journal for Numerical Methods in Fluids, 16, 489-505, https://doi.org/10.1002/fld.1650160604, 1993.

Amini, J.: A method for generating floodplain maps using IKONOS images and DEMs, International Journal of Remote Sensing, 31, 24412456, https://doi.org/10.1080/01431160902929230, 2010.

Ávila, A., Justino, F., Wilson, A., Bromwich, D., and Amorim, M.: Recent precipitation trends, flash floods and landslides in southern Brazil, Environmental Research Letters, 11, https://doi.org/10.1088/1748-9326/11/11/114029, 2016.

Badrinarayanan, V., Handa, A., and Cipolla, R.: Segnet: A deep convolutional encoder-decoder architecture for robust semantic pixel-wise labelling, arXiv preprint arXiv:1505.07293, 2015.

Balestriero, R., Pesenti, J., and LeCun, Y.: Learning in High Dimension Always Amounts to Extrapolation, arXiv preprint arXiv:2110.09485, 2021.

Bates, P. D. and De Roo, A. P.: A simple raster-based model for flood inundation simulation, Journal of Hydrology, 236, 54-77, https://doi.org/10.1016/S0022-1694(00)00278-X, 2000.

Battaglia, P. W., Hamrick, J. B., Bapst, V., Sanchez-Gonzalez, A., Zambaldi, V., Malinowski, M., Tacchetti, A., Raposo, D., Santoro, A., Faulkner, R., Gulcehre, C., Song, F., Ballard, A., Gilmer, J., Dahl, G., Vaswani, A., Allen, K., Nash, C., Langston, V., Dyer, C., Heess, N., Wierstra, D., Kohli, P., Botvinick, M., Vinyals, O., Li, Y., and Pascanu, R.: Relational inductive biases, deep learning, and graph networks, arXiv, pp. 1-40, 2018.

Berkhahn, S., Fuchs, L., and Neuweiler, I.: An ensemble neural network model for real-time prediction of urban floods, Journal of Hydrology, $575,743-754,2019$.

Bobée, B. and Rasmussen, P. F.: Recent advances in flood frequency analysis, Reviews of Geophysics, 33, 1111-1116, 1995.

Bodnar, C., Frasca, F., Otter, N., Wang, Y. G., Liò, P., Montúfar, G., and Bronstein, M.: Weisfeiler and lehman go cellular: Cw networks, arXiv preprint arXiv:2106.12575, 2021.

Bomers, A., Schielen, R. M., and Hulscher, S. J.: The influence of grid shape and grid size on hydraulic river modelling performance, Environmental Fluid Mechanics, 19, 1273-1294, https://doi.org/10.1007/s10652-019-09670-4, 2019.

Bowes, B. D., Tavakoli, A., Wang, C., Heydarian, A., Behl, M., Beling, P. A., and Goodall, J. L.: Flood mitigation in coastal urban catchments using real-time stormwater infrastructure control and reinforcement learning, Journal of Hydroinformatics, 23, 529-547, 2021.

Bronstein, M. M., Bruna, J., Lecun, Y., Szlam, A., and Vandergheynst, P.: Geometric Deep Learning: Going beyond Euclidean data, IEEE Signal Processing Magazine, 34, 18-42, https://doi.org/10.1109/MSP.2017.2693418, 2017.

Bronstein, M. M., Bruna, J., Cohen, T., and Veličković, P.: Geometric deep learning: Grids, groups, graphs, geodesics, and gauges, arXiv preprint arXiv:2104.13478, 2021. 
Chang, D.-L., Yang, S.-H., Hsieh, S.-L., Wang, H.-J., and Yeh, K.-C.: Artificial intelligence methodologies applied to prompt pluvial flood estimation and prediction, Water (Switzerland), 12, 2020.

Chang, L.-C., Shen, H.-Y., Wang, Y.-F., Huang, J.-Y., and Lin, Y.-T.: Clustering-based hybrid inundation model for forecasting flood inundation depths, Journal of Hydrology, 385, 257-268, https://doi.org/https://doi.org/10.1016/j.jhydrol.2010.02.028, 2010.

Chen, J., Huang, G., and Chen, W.: Towards better flood risk management: Assessing flood risk and investigating the potential mechanism based on machine learning models, https://doi.org/10.1016/j.jenvman.2021.112810, 2021.

Cho, K., van Merrienboer, B., Bahdanau, D., and Bengio, Y.: On the Properties of Neural Machine Translation: Encoder-Decoder Approaches, 2014.

Chu, H., Wu, W., Wang, Q., Nathan, R., and Wei, J.: An ANN-based emulation modelling framework for flood inundation modelling: Application, challenges and future directions, Environmental Modelling and Software, 124, 2020.

Cian, F., Marconcini, M., and Ceccato, P.: Normalized Difference Flood Index for rapid flood mapping: Taking advantage of EO big data, Remote Sensing of Environment, 209, 712-730, https://doi.org/https://doi.org/10.1016/j.rse.2018.03.006, 2018.

Costabile, P., Macchione, F., Natale, L., and Petaccia, G.: Flood mapping using LIDAR DEM. Limitations of the 1-D modeling highlighted by the 2-D approach, Natural Hazards, 77, 181-204, https://doi.org/10.1007/s11069-015-1606-0, 2015.

815 Costabile, P., Costanzo, C., and Macchione, F.: Performances and limitations of the diffusive approximation of the 2-d shallow water equations for flood simulation in urban and rural areas, Applied Numerical Mathematics, 116, 141-156, https://doi.org/10.1016/j.apnum.2016.07.003, 2017.

Costache, R., Ngo, P., and Bui, D.: Novel ensembles of deep learning neural network and statistical learning for flash-flood susceptibility mapping, Water (Switzerland), 12, 2020.

Cunge, J., Holly, F., and Verwey, A.: Practical aspects of computational river hydraulics, Pitman, 1980.

Damianou, A. and Lawrence, N. D.: Deep gaussian processes, in: Artificial intelligence and statistics, pp. 207-215, PMLR, 2013.

Darabi, H., Rahmati, O., Naghibi, S., Mohammadi, F., Ahmadisharaf, E., Kalantari, Z., Torabi Haghighi, A., Soleimanpour, S., Tiefenbacher, J., and Tien Bui, D.: Development of a novel hybrid multi-boosting neural network model for spatial prediction of urban flood, Geocarto International, 2021.

de Brito, M. M. and Evers, M.: Multi-criteria decision-making for flood risk management: a survey of the current state of the art, Natural Hazards and Earth System Sciences, 16, 1019-1033, https://doi.org/10.5194/nhess-16-1019-2016, 2016.

De Haan, P., Weiler, M., Cohen, T., and Welling, M.: Gauge equivariant mesh CNNs anisotropic convolutions on geometric graphs, arXiv, 2020.

de Moel, H., van Alphen, J., and Aerts, J. C. J. H.: Flood maps in Europe ndash; methods, availability and use, Natural Hazards and Earth System Sciences, 9, 289-301, https://doi.org/10.5194/nhess-9-289-2009, 2009.

de Moel, H., Jongman, B., Kreibich, H., Merz, B., Penning-Rowsell, E., and Ward, P. J.: Flood risk assessments at different spatial scales, Mitigation and Adaptation Strategies for Global Change, 20, 865-890, 2015.

Destro, E., Amponsah, W., Nikolopoulos, E. I., Marchi, L., Marra, F., Zoccatelli, D., and Borga, M.: Coupled prediction of flash flood response and debris flow occurrence: Application on an alpine extreme flood event, Journal of Hydrology, 558, 225-237, https://doi.org/10.1016/j.jhydrol.2018.01.021, 2018.

Di Baldassarre, G., Schumann, G., Bates, P. D., Freer, J. E., and Beven, K. J.: Flood-plain mapping: a critical discussion of deterministic and probabilistic approaches, Hydrological Sciences Journal-Journal des Sciences Hydrologiques, 55, 364-376, 2010. 
Domeneghetti, A., Vorogushyn, S., Castellarin, A., Merz, B., and Brath, A.: Probabilistic flood hazard mapping: effects of uncertain boundary conditions, Hydrology and Earth System Sciences, 17, 3127-3140, 2013.

Dong, S., Yu, T., Farahmand, H., and Mostafavi, A.: A hybrid deep learning model for predictive flood warning and situation awareness using channel network sensors data, Computer-Aided Civil and Infrastructure Engineering, 36, 402-420, https://doi.org/https://doi.org/10.1111/mice.12629, 2021.

Dottori, F. and Todini, E.: A 2D Flood Inundation Model Based on Cellular Automata Approach, XVIII International Conference on Water Resources, pp. 1-8, 2010.

Dottori, F., Alfieri, L., Bianchi, A., Skoien, J., and Salamon, P.: A new dataset of river flood hazard maps for Europe and the Mediterranean Basin region, Earth System Science Data Discussions, 2021, 1-35, https://doi.org/10.5194/essd-2020-313, 2021.

Ebli, S., Defferrard, M., and Spreemann, G.: Simplicial Neural Networks, arXiv preprint arXiv:2010.03633, 2020.

European Union: Directive 2007/60/EC of the European Counil and European Parliment of 23 October 2007 on the assessment and management of flood risks, Official Journal of the European Union, pp. 27-34, http://eur-lex.europa.eu/legal-content/EN/TXT/PDF/?uri=CELEX: 32007L0060\&from=EN, 2007.

Fang, Z., Wang, Y., Peng, L., and Hong, H.: Predicting flood susceptibility using LSTM neural networks, Journal of Hydrology, p. 125734, https://doi.org/10.1016/j.jhydrol.2020.125734, 2020a.

Fang, Z., Yang, T., and Jin, Y.: DeepStreet: A deep learning powered urban street network generation module, arXiv preprint arXiv:2010.04365, 2020b.

Feng, Y., Brenner, C., and Sester, M.: Flood severity mapping from Volunteered Geographic Information by interpreting water level from images containing people: A case study of Hurricane Harvey, ISPRS Journal of Photogrammetry and Remote Sensing, 169, 301-319, 2020.

Ferraro, D., Costabile, P., Costanzo, C., Petaccia, G., and Macchione, F.: A spectral analysis approach for the a priori generation of computational grids in the 2-D hydrodynamic-based runoff simulations at a basin scale, Journal of Hydrology, 582, 124508, https://doi.org/10.1016/j.jhydrol.2019.124508, 2020.

Ferreira, L. A., Fonseca, A. R., Lima, N. Z., Mesquita, R. C., and Salgado, G. C.: Graphical interface for electromagnetic problem solving using meshless methods, Journal of Microwaves, Optoelectronics and Electromagnetic Applications (JMOe), 14, 2015.

Finaud-Guyot, P., Delenne, C., Guinot, V., and Llovel, C.: 1D-2D coupling for river flow modeling, Comptes Rendus - Mecanique, 339, 226-234, https://doi.org/10.1016/j.crme.2011.02.001, 2011.

Gama, F., Isufi, E., Leus, G., and Ribeiro, A.: Graphs, Convolutions, and Neural Networks, 2020.

Gebrehiwot, A., Hashemi-Beni, L., Thompson, G., Kordjamshidi, P., and Langan, T. E.: Deep convolutional neural network for flood extent mapping using unmanned aerial vehicles data, Sensors (Switzerland), 19, https://doi.org/10.3390/s19071486, 2019.

Glenis, V., McGough, A. S., Kutija, V., Kilsby, C., and Woodman, S.: Flood modelling for cities using Cloud computing, Journal of Cloud Computing: Advances, Systems and Applications, 2, 1-14, 2013.

870 Goodfellow, I.: Nips 2016 tutorial: Generative adversarial networks, arXiv preprint arXiv:1701.00160, 2016.

Goodfellow, I., Pouget-Abadie, J., Mirza, M., Xu, B., Warde-Farley, D., Ozair, S., Courville, A., and Bengio, Y.: Generative adversarial nets, Advances in neural information processing systems, 27, 2014.

Goodfellow, I., Bengio, Y., and Courville, A.: Deep Learning, MIT Press, http://www.deeplearningbook.org, 2016.

Guo, Z., Leitão, J. P., Simões, N. E., and Moosavi, V.: Data-driven flood emulation: Speeding up urban flood predictions by deep convolutional neural networks, Journal of Flood Risk Management, pp. 1-14, https://doi.org/10.1111/jfr3.12684, 2020. 
Hajij, M., Istvan, K., and Zamzmi, G.: Cell complex neural networks, arXiv preprint arXiv:2010.00743, 2020.

Hashemi-Beni, L. and Gebrehiwot, A.: Flood Extent Mapping: An Integrated Method Using Deep Learning and Region Growing Using UAV Optical Data, IEEE Journal of Selected Topics in Applied Earth Observations and Remote Sensing, 14, 2127-2135, 2021.

Hess, L., Melack, J., Filoso, S., and Wang, Y.: Delineation of inundated area and vegetation along the Amazon floodplain with the SIR-C synthetic aperture radar, IEEE Transactions on Geoscience and Remote Sensing, 33, 896-904, https://doi.org/10.1109/36.406675, 1995.

Hochreiter, S. and Schmidhuber, J.: Long short-term memory, Neural computation, 9, 1735-1780, 1997.

Horritt, M. S. and Bates, P. D.: Evaluation of 1D and 2D numerical models for predicting river flood inundation, Journal of Hydrology, 268, 87-99, https://doi.org/10.1016/S0022-1694(02)00121-X, 2002.

Hou, J., Li, X., Bai, G., Wang, X., Zhang, Z., Yang, L., Du, Y., Ma, Y., Fu, D., and Zhang, X.: A deep learning technique based flood propagation experiment, Journal of Flood Risk Management, 2021.

Hu, R., Fang, F., Pain, C., and Navon, I.: Rapid spatio-temporal flood prediction and uncertainty quantification using a deep learning method, Journal of Hydrology, 575, 911-920, https://doi.org/https://doi.org/10.1016/j.jhydrol.2019.05.087, 2019.

Huang, P.-C., Hsu, K.-L., and Lee, K.: Improvement of Two-Dimensional Flow-Depth Prediction Based on Neural Network Models By Preprocessing Hydrological and Geomorphological Data, Water Resources Management, 35, 1079-1100, $2021 \mathrm{a}$.

Huang, P. C., Hsu, K. L., and Lee, K. T.: Improvement of Two-Dimensional Flow-Depth Prediction Based on Neural Network Models By Preprocessing Hydrological and Geomorphological Data, Water Resources Management, pp. 1079-1100, https://doi.org/10.1007/s11269021-02776-9, 2021b.

Ichim, L. and Popescu, D.: Segmentation of vegetation and flood from aerial images based on decision fusion of neural networks, Remote Sensing, 12, 2020.

Ireland, G., Volpi, M., and Petropoulos, G. P.: Examining the Capability of Supervised Machine Learning Classifiers in Extracting Flooded Areas from Landsat TM Imagery: A Case Study from a Mediterranean Flood, Remote Sensing, 7, 3372-3399, https://doi.org/10.3390/rs70303372, 2015.

Isufi, E., Gama, F., and Ribeiro, A.: EdgeNets: Edge varying graph neural networks, IEEE Transactions on Pattern Analysis and Machine Intelligence, 2021.

Jafari, N., Li, X., Chen, Q., Le, C.-Y., Betzer, L., and Liang, Y.: Real-time water level monitoring using live cameras and computer vision techniques, Computers and Geosciences, 147, 2021.

Jahangir, M., Mousavi Reineh, S., and Abolghasemi, M.: Spatial predication of flood zonation mapping in Kan River Basin, Iran, using artificial neural network algorithm, Weather and Climate Extremes, 25, 2019.

Jonkman, S. and Vrijling, J.: Loss of life due to floods, Journal of Flood Risk Management, 1, 43-56, https://doi.org/10.1111/j.1753318x.2008.00006.x, 2008.

Kabir, S., Patidar, S., Xia, X., Liang, Q., Neal, J., and Pender, G.: A deep convolutional neural network model for rapid prediction of fluvial flood inundation, Journal of Hydrology, 590, 125 481, https://doi.org/10.1016/j.jhydrol.2020.125481, 2020.

Kalantar, B., Ueda, N., Saeidi, V., Janizadeh, S., Shabani, F., Ahmadi, K., and Shabani, F.: Deep Neural Network Utilizing Remote Sensing Datasets for Flood Hazard Susceptibility Mapping in Brisbane, Australia, Remote Sensing, 13, https://doi.org/10.3390/rs13132638, 2021.

910 Kang, W., Xiang, Y., Wang, F., Wan, L., and You, H.: Flood Detection in Gaofen-3 SAR Images via Fully Convolutional Networks, Sensors, 18, https://doi.org/10.3390/s18092915, 2018.

Kao, I. F., Liou, J. Y., Lee, M. H., and Chang, F. J.: Fusing stacked autoencoder and long short-term memory for regional multistep-ahead flood inundation forecasts, Journal of Hydrology, p. 126371, https://doi.org/10.1016/j.jhydrol.2021.126371, 2021. 
Kazakis, N., Kougias, I., and Patsialis, T.: Assessment of flood hazard areas at a regional scale using an index-based approach and Analytical Hierarchy Process: Application in Rhodope-Evros region, Greece, Science of The Total Environment, 538, 555-563, https://doi.org/https://doi.org/10.1016/j.scitotenv.2015.08.055, 2015.

Khoirunisa, N., Ku, C.-Y., and Liu, C.-Y.: A GIS-based artificial neural network model for flood susceptibility assessment, International Journal of Environmental Research and Public Health, 18, 1-20, 2021.

Khosravi, K., Panahi, M., Golkarian, A., Keesstra, S. D., and Saco, P. M.: Convolutional neural network approach for spatial prediction of flood hazard at national scale of Iran, Journal of Hydrology, 591, 125 552, https://doi.org/10.1016/j.jhydrol.2020.125552, 2020.

Kia, M. B., Pirasteh, S., Pradhan, B., Mahmud, A. R., Sulaiman, W. N. A., and Moradi, A.: An artificial neural network model for flood simulation using GIS: Johor River Basin, Malaysia, Environmental earth sciences, 67, 251-264, 2012.

Kingma, D. P. and Welling, M.: Auto-encoding variational bayes, arXiv preprint arXiv:1312.6114, 2013.

Kourgialas, N. N. and Karatzas, G. P.: A national scale flood hazard mapping methodology: The case of Greece - Protection and adaptation policy approaches, Science of The Total Environment, 601-602, 441-452, https://doi.org/https://doi.org/10.1016/j.scitotenv.2017.05.197, 2017.

Kovachki, N., Li, Z., Liu, B., Azizzadenesheli, K., Bhattacharya, K., Stuart, A., and Anandkumar, A.: Neural operator: Learning maps between function spaces, arXiv preprint arXiv:2108.08481, 2021.

Kratzert, F., Herrnegger, M., Klotz, D., Hochreiter, S., and Klambauer, G.: NeuralHydrology - Interpreting LSTMs in Hydrology, Lecture Notes in Computer Science (including subseries Lecture Notes in Artificial Intelligence and Lecture Notes in Bioinformatics), 11700 LNCS, 347-362, https://doi.org/10.1007/978-3-030-28954-6_19, 2019a.

Kratzert, F., Klotz, D., Herrnegger, M., Sampson, A. K., Hochreiter, S., and Nearing, G. S.: Toward Improved Predictions in Ungauged Basins: Exploiting the Power of Machine Learning, Water Resources Research, 55, 11344-11354, https://doi.org/10.1029/2019WR026065, 2019 b.

Kummu, M., De Moel, H., Ward, P. J., and Varis, O.: How close do we live to water? A global analysis of population distance to freshwater bodies, PloS one, 6, e20 578, 2011.

Lane, S. N., Bradbrook, K. F., Richards, K. S., Biron, P. A., and Roy, A. G.: The application of computational fluid dynamics to natural river channels: Three-dimensional versus two-dimensional approaches, Geomorphology, 29, 1-20, https://doi.org/10.1016/S0169555X(99)00003-3, 1999.

940 Leandro, J., Chen, A. S., Djordjević, S., and Savić, D. A.: Comparison of 1D/1D and 1D/2D coupled (sewer/surface) hydraulic models for urban flood simulation, Journal of hydraulic engineering, 135, 495-504, 2009.

LeCun, Y., Bengio, Y., et al.: Convolutional networks for images, speech, and time series, The handbook of brain theory and neural networks, $3361,1995,1995$.

LeCun, Y., Bengio, Y., and Hinton, G.: Deep learning, nature, 521, 436-444, 2015.

945 Lendering, K., Jonkman, S., and Kok, M.: Effectiveness of emergency measures for flood prevention, Journal of Flood Risk Management, 9 , 320-334, 2016.

Li, L., Chen, Y., Xu, T., Liu, R., Shi, K., and Huang, C.: Super-resolution mapping of wetland inundation from remote sensing imagery based on integration of back-propagation neural network and genetic algorithm, Remote Sensing of Environment, 164, 142-154, https://doi.org/https://doi.org/10.1016/j.rse.2015.04.009, 2015. 

ticle swarm optimization for enhancing sub-pixel mapping of flood inundation in river basins, Remote Sensing Letters, 7, 631-640, https://doi.org/10.1080/2150704X.2016.1177238, 2016a.

Li, L., Xu, T., and Chen, Y.: Improved urban flooding mapping from remote sensing images using generalized regression neural networkbased super-resolution algorithm, Remote Sensing, 8, https://doi.org/10.3390/rs8080625, 2016b.

Li, Z., Kovachki, N., Azizzadenesheli, K., Liu, B., Bhattacharya, K., Stuart, A., and Anandkumar, A.: Fourier Neural Operator for Parametric Partial Differential Equations, arXiv preprint arXiv:2010.08895, 2021.

Lin, L., Di, L., Yu, E. G., Kang, L., Shrestha, R., Rahman, M. S., Tang, J., Deng, M., Sun, Z., Zhang, C., et al.: A review of remote sensing in flood assessment, in: 2016 Fifth International Conference on Agro-Geoinformatics (Agro-Geoinformatics), pp. 1-4, IEEE, 2016.

Lin, Q., Leandro, J., Gerber, S., and Disse, M.: Multistep flood inundation forecasts with resilient backpropagation neural networks: Kulmbach case study, Water (Switzerland), 12, 2020a.

Lin, Q., Leandro, J., Wu, W., Bhola, P., and Disse, M.: Prediction of Maximum Flood Inundation Extents With Resilient Backpropagation Neural Network: Case Study of Kulmbach, Frontiers in Earth Science, 8, 2020b.

Lino, M., Cantwell, C., Bharath, A. A., and Fotiadis, S.: Simulating Continuum Mechanics with Multi-Scale Graph Neural Networks, arXiv preprint arXiv:2106.04900, 2021.

Liu, B., Li, X., and Zheng, G.: Coastal Inundation Mapping From Bitemporal and Dual-Polarization SAR Imagery Based on Deep Convolutional Neural Networks, Journal of Geophysical Research: Oceans, 124, 9101-9113, 2019.

Liu, M.: Optimal number of trials for Monte Carlo simulation, VRC-Valuation Research Report, 2017.

Lu, L., Jin, P., and Karniadakis, G. E.: DeepONet: Learning nonlinear operators for identifying differential equations based on the universal approximation theorem of operators, CoRR, abs/1910.03193, http://arxiv.org/abs/1910.03193, 2019.

Lütjens, B., Leshchinskiy, B., Requena-Mesa, C., Chishtie, F., Díaz-Rodriguez, N., Boulais, O., Piña, A., Newman, D., Lavin, A., Gal, Y., et al.: Physics-informed gans for coastal flood visualization, arXiv preprint arXiv:2010.08103, 2020.

Lütjens, B., Leshchinskiy, B., Requena-Mesa, C., Chishtie, F., Díaz-Rodríguez, N., Boulais, O., Sankaranarayanan, A., Piña, A., Gal, Y., Raïssi, C., et al.: Physically-Consistent Generative Adversarial Networks for Coastal Flood Visualization, arXiv preprint arXiv:2104.04785, 2021.

Löwe, R., Böhm, J., Jensen, D. G., Leandro, J., and Rasmussen, S. H.: U-FLOOD - Topographic deep learning for predicting urban pluvial flood water depth, Journal of Hydrology, 603, 126 898, https://doi.org/https://doi.org/10.1016/j.jhydrol.2021.126898, 2021.

Ma, X., Hong, Y., Song, Y., and Chen, Y.: A super-resolution convolutional-neural-network-based approach for subpixel mapping of hyperspectral images, IEEE Journal of Selected Topics in Applied Earth Observations and Remote Sensing, 12, 4930-4939, 2019.

Mahmoud, S. H. and Gan, T. Y.: Multi-criteria approach to develop flood susceptibility maps in arid regions of Middle East, Journal of Cleaner Production, 196, 216-229, https://doi.org/https://doi.org/10.1016/j.jclepro.2018.06.047, 2018.

Manavalan, R.: SAR image analysis techniques for flood area mapping-literature survey, Earth Science Informatics, 10, 1-14, 2017.

Manjusree, P., Kumar, L. P., Bhatt, C. M., Rao, G. S., and Bhanumurthy, V.: Optimization of threshold ranges for rapid flood inundation mapping by evaluating backscatter profiles of high incidence angle SAR images, International Journal of Disaster Risk Science, 3, 113$122,2012$.

Mao, Z., Jagtap, A. D., and Karniadakis, G. E.: Physics-informed neural networks for high-speed flows, Computer Methods in Applied Mechanics and Engineering, 360, 112 789, https://doi.org/https://doi.org/10.1016/j.cma.2019.112789, 2020. 
Martinis, S., Twele, A., and Voigt, S.: Towards operational near real-time flood detection using a split-based automatic thresholding procedure on high resolution TerraSAR-X data, Natural Hazards and Earth System Sciences, 9, 303-314, https://doi.org/10.5194/nhess-9-303-2009, 2009.

Masson-Delmotte, V., P, Z., A, P., S L, C., C, P., S, B., N, C., Y, C., L, G., M I, G., M, H., K, L., E, L., J B R, M., T K, M., T, W., O, Y., R, Y., and B, Z.: Climate Change 2021: The Physical Science Basis. Contribution of Working Group I to the Sixth Assessment Report of the Intergovernmental Panel on Climate Change, Cambridge University Press. In Press., 2021.

Mavriplis, D.: Unstructured grid techniques, Annual Review of Fluid Mechanics, 29, 473-514, 1997.

Ming, X., Liang, Q., Xia, X., Li, D., and Fowler, H. J.: Real-Time Flood Forecasting Based on a High-Performance 2-D Hydrodynamic Model and Numerical Weather Predictions, Water Resources Research, 56, https://doi.org/10.1029/2019WR025583, 2020.

Mitchell, T.: Machine Learning, 1997.

Mosavi, A., Ozturk, P., and Chau, K.-w.: Flood Prediction Using Machine Learning Models: Literature Review, Water, 10, https://doi.org/10.3390/w10111536, 2018.

Muñoz, D., Muñoz, P., Moftakhari, H., and Moradkhani, H.: From local to regional compound flood mapping with deep learning and data fusion techniques, Science of the Total Environment, 782, https://doi.org/10.1016/j.scitotenv.2021.146927, 2021.

Nemni, E., Bullock, J., Belabbes, S., and Bromley, L.: Fully Convolutional Neural Network for Rapid Flood Segmentation in Synthetic Aperture Radar Imagery, Remote Sensing, 12, https://doi.org/10.3390/rs12162532, 2020.

Neumann, B., Vafeidis, A. T., Zimmermann, J., and Nicholls, R. J.: Future coastal population growth and exposure to sea-level rise and coastal flooding-a global assessment, PloS one, 10, e 0118 571, 2015.

Ngo, P. T. T., Hoang, N. D., Pradhan, B., Nguyen, Q. K., Tran, X. T., Nguyen, Q. M., Nguyen, V. N., Samui, P., and Bui, D. T.: A novel hybrid swarm optimized multilayer neural network for spatial prediction of flash floods in tropical areas using sentinel-1 SAR imagery and geospatial data, Sensors (Switzerland), 18, https://doi.org/10.3390/s18113704, 2018.

Nogueira, K., Fadel, S. G., Dourado, I. C., De O. Werneck, R., Muñoz, J. A., Penatti, O. A., Calumby, R. T., Li, L. T., Dos Santos, J. A., and Da S. Torres, R.: Exploiting ConvNet diversity for flooding identification, arXiv, 15, 1446-1450, 2017.

Observatory, D. F.: Space-based Measurement, Mapping, and Modeling of Surface Water, https://floodobservatory.colorado.edu/, accessed: 2021-11-11.

Oord, A. v. d., Dieleman, S., Zen, H., Simonyan, K., Vinyals, O., Graves, A., Kalchbrenner, N., Senior, A., and Kavukcuoglu, K.: Wavenet: A generative model for raw audio, arXiv preprint arXiv:1609.03499, 2016.

Panahi, M., Jaafari, A., Shirzadi, A., Shahabi, H., Rahmati, O., Omidvar, E., Lee, S., and Bui, D.: Deep learning neural networks for spatially explicit prediction of flash flood probability, Geoscience Frontiers, 12, 2021.

Papaioannou, G., Vasiliades, L., Loukas, A., and Aronica, G. T.: Probabilistic flood inundation mapping at ungauged streams due to roughness coefficient uncertainty in hydraulic modelling, Advances in Geosciences, 44, 23-34, 2017.

Peng, B., Meng, Z., Huang, Q., and Wang, C.: Patch Similarity Convolutional Neural Network for Urban Flood Extent Mapping Using Bi-Temporal Satellite Multispectral Imagery, Remote Sensing, 11, https://doi.org/10.3390/rs11212492, 2019.

Pfaff, T., Fortunato, M., Sanchez-Gonzalez, A., and Battaglia, P. W.: Learning Mesh-Based Simulation with Graph Networks, International Conference on Learning Representations (ICLR), 2021.

Popa, M., Peptenatu, D., Draghici, C., and Diaconu, D.: Flood hazard mapping using the flood and Flash-Flood Potential Index in the Buzau River catchment, Romania, Water (Switzerland), 11, 2019. 
Prestininzi, P.: Suitability of the diffusive model for dam break simulation: Application to a CADAM experiment, Journal of Hydrology, 361, $172-185,2008$

Raissi, M., Perdikaris, P., and Karniadakis, G. E.: Physics-informed neural networks: A deep learning framework for solving forward and inverse problems involving nonlinear partial differential equations, Journal of Computational Physics, 378, 686-707, https://doi.org/10.1016/j.jcp.2018.10.045, 2019.

Rasmussen, C. E.: Gaussian processes in machine learning, in: Summer school on machine learning, pp. 63-71, Springer, 2003.

Ravuri, S., Lenc, K., Willson, M., Kangin, D., Lam, R., Mirowski, P., Fitzsimons, M., Athanassiadou, M., Kashem, S., Madge, S., et al.: Skillful Precipitation Nowcasting using Deep Generative Models of Radar, arXiv preprint arXiv:2104.00954, 2021.

Rossi, C., Acerbo, F., Ylinen, K., Juga, I., Nurmi, P., Bosca, A., Tarasconi, F., Cristoforetti, M., and Alikadic, A.: Early detection and information extraction for weather-induced floods using social media streams, International Journal of Disaster Risk Reduction, 30, 145157, https://doi.org/https://doi.org/10.1016/j.ijdrr.2018.03.002, communicating High Impact Weather: Improving warnings and decision making processes, 2018.

Rumelhart, D. E., Hinton, G. E., and Williams, R. J.: Learning representations by back-propagating errors, nature, 323, 533-536, 1986.

Sarker, C., Mejias, L., Maire, F., and Woodley, A.: Flood Mapping with Convolutional Neural Networks Using Spatio-Contextual Pixel Information, Remote Sensing, 11, https://doi.org/10.3390/rs11192331, 2019.

Schmidt, V., Luccioni, A., Mukkavilli, S. K., Balasooriya, N., Sankaran, K., Chayes, J., and Bengio, Y.: Visualizing the consequences of climate change using cycle-consistent adversarial networks, arXiv preprint arXiv:1905.03709, 2019.

Serinaldi, F., Loecker, F., Kilsby, C. G., and Bast, H.: Flood propagation and duration in large river basins: a data-driven analysis for reinsurance purposes, Natural Hazards, 94, 71-92, https://doi.org/10.1007/s11069-018-3374-0, 2018.

Shi, X., Chen, Z., Wang, H., Yeung, D. Y., Wong, W. K., and Woo, W. C.: Convolutional LSTM network: A machine learning approach for precipitation nowcasting, Advances in Neural Information Processing Systems, 2015-Janua, 802-810, 2015.

Sikorska, A. E., Viviroli, D., and Seibert, J.: Flood-type classification in mountainous catchments using crisp and fuzzy decision trees, Journal of the American Water Resources Association, 5, 2-2, https://doi.org/10.1111/j.1752-1688.1969.tb04897.x, 2015.

Sit, M., Demiray, B. Z., Xiang, Z., Ewing, G. J., Sermet, Y., and Demir, I.: A comprehensive review of deep learning applications in hydrology and water resources, Water Science and Technology, 82, 2635-2670, https://doi.org/10.2166/wst.2020.369, 2020.

Sridharan, B., Bates, P. D., Sen, D., and Kuiry, S. N.: Local-inertial shallow water model on unstructured triangular grids, Advances in Water Resources, 152, 103 930, https://doi.org/10.1016/j.advwatres.2021.103930, 2021.

Taormina, R. and Galelli, S.: Deep-learning approach to the detection and localization of cyber-physical attacks on water distribution systems, Journal of Water Resources Planning and Management, 144, 04018 065, 2018.

Tayefi, V., Lane, S., Hardy, R., and Yu, D.: A comparison of one-and two-dimensional approaches to modelling flood inundation over complex upland floodplains, Hydrological Processes: An International Journal, 21, 3190-3202, 2007.

Tehrany, M. S., Lee, M.-J., Pradhan, B., Jebur, M. N., and Lee, S.: Flood susceptibility mapping using integrated bivariate and multivariate statistical models, Environmental earth sciences, 72, 4001-4015, 2014.

Teng, J., Jakeman, A. J., Vaze, J., Croke, B. F., Dutta, D., and Kim, S.: Flood inundation modelling: A review of methods, recent advances and uncertainty analysis, Environmental Modelling and Software, 90, 201-216, https://doi.org/10.1016/j.envsoft.2017.01.006, 2017.

Tien, D., Hoang, N.-d., Martínez-álvarez, F., Ngo, P.-t. T., Viet, P., Dat, T., Samui, P., and Costache, R.: A novel deep learning neural network approach for predicting flash flood susceptibility : A case study at a high frequency tropical storm area, Science of the Total Environment, 701, 134 413, https://doi.org/10.1016/j.scitotenv.2019.134413, 2020. 
Vacondio, R., Rogers, B., Stansby, P., and Mignosa, P.: SPH modeling of shallow flow with open boundaries for practical flood simulation, Journal of Hydraulic Engineering, 138, 530-541, 2012.

Vandenberg-Rodes, A., Moftakhari, H. R., AghaKouchak, A., Shahbaba, B., Sanders, B. F., and Matthew, R. A.: Projecting nuisance flooding in a warming climate using generalized linear models and Gaussian processes, Journal of Geophysical Research: Oceans, 121, 8008-8020, https://doi.org/https://doi.org/10.1002/2016JC012084, 2016.

Wang, Y., Fang, Z., Hong, H., and Peng, L.: Flood susceptibility mapping using convolutional neural network frameworks, Journal of Hydrology, 582, 124 482, https://doi.org/10.1016/j.jhydrol.2019.124482, 2020.

Wu, Z., Pan, S., Chen, F., Long, G., Zhang, C., and Yu, P. S.: A Comprehensive Survey on Graph Neural Networks, IEEE Transactions on Neural Networks and Learning Systems, 32, 4-24, https://doi.org/10.1109/TNNLS.2020.2978386, 2021.

Yakti, B. P., Adityawan, M. B., Farid, M., Suryadi, Y., Nugroho, J., and Hadihardaja, I. K.: 2D modeling of flood propagation due to the failure of way Ela natural dam, in: MATEC Web of Conferences, vol. 147, EDP Sciences, 2018.

Yang, M., Isufi, E., and Leus, G.: Simplicial Convolutional Neural Networks, arXiv preprint arXiv:2110.02585, 2021.

Yang, W., Zhang, X., Tian, Y., Wang, W., Xue, J.-H., and Liao, Q.: Deep learning for single image super-resolution: A brief review, IEEE Transactions on Multimedia, 21, 3106-3121, 2019a.

Yang, X. I. A., Zafar, S., Wang, J.-X., and Xiao, H.: Predictive large-eddy-simulation wall modeling via physics-informed neural networks, Phys. Rev. Fluids, 4, 034 602, https://doi.org/10.1103/PhysRevFluids.4.034602, 2019b.

Yi, Y., Zhang, Z., Zhang, W., Jia, H., and Zhang, J.: Landslide susceptibility mapping using multiscale sampling strategy and convolutional neural network: A case study in Jiuzhaigou region, Catena, 195, 104 851, 2020.

Youssef, A. M., Pradhan, B., and Sefry, S. A.: Flash flood susceptibility assessment in Jeddah city (Kingdom of Saudi Arabia) using bivariate and multivariate statistical models, Environmental Earth Sciences, 75, 12, 2016.

Zhang, S., Xia, Z., Yuan, R., and Jiang, X.: Parallel computation of a dam-break flow model using OpenMP on a multi-core computer, Journal of hydrology, 512, 126-133, 2014.

Zhao, G., Bates, P., Neal, J., and Pang, B.: Design flood estimation for global river networks based on machine learning models, Hydrology and Earth System Sciences Discussions, 2015, 1-25, https://doi.org/10.5194/hess-2020-594, 2020a.

Zhao, G., Pang, B., Xu, Z., Peng, D., and Zuo, D.: Urban flood susceptibility assessment based on convolutional neural networks, Journal of Hydrology, 590, 125 235, https://doi.org/10.1016/j.jhydrol.2020.125235, 2020b.

Zhao, G., Balstrøm, T., Mark, O., and Jensen, M. B.: Multi-Scale Target-Specified Sub-Model Approach for Fast Large-Scale HighResolution 2D Urban Flood Modelling, Water, 13, https://doi.org/10.3390/w13030259, 2021a.

Zhao, G., Pang, B., Xu, Z., Cui, L., Wang, J., Zuo, D., and Peng, D.: Improving urban flood susceptibility mapping using transfer learning, Journal of Hydrology, 602, 126 777, https://doi.org/https://doi.org/10.1016/j.jhydrol.2021.126777, $2021 \mathrm{~b}$.

Zhou, Y., Wu, C., Li, Z., Cao, C., Ye, Y., Saragih, J., Li, H., and Sheikh, Y.: Fully Convolutional Mesh Autoencoder using Efficient Spatially Varying Kernels, arXiv, 2020.

Zhou, Y., Wu, W., Nathan, R., and Wang, Q. J.: A rapid flood inundation modelling framework using deep learning with spatial reduction and reconstruction, Environmental Modelling and Software, 143, 105 112, https://doi.org/https://doi.org/10.1016/j.envsoft.2021.105112, 2021.

Zounemat-Kermani, M., Matta, E., Cominola, A., Xia, X., Zhang, Q., Liang, Q., and Hinkelmann, R.: Neurocomputing in surface water hydrology and hydraulics: A review of two decades retrospective, current status and future prospects, Journal of Hydrology, 588, 125085 , https://doi.org/https://doi.org/10.1016/j.jhydrol.2020.125085, 2020. 